\title{
Study of rare $b$ decays with the DELPHI detector at LEP
}

\author{
DELPHI Collaboration
}

\begin{abstract}
Rare decays of beauty particles were studied in several charmless modes using the data collected with the DELPHI detector at LEP from 1991 to 1994. These decays are mediated by both tree level $b \rightarrow u$ and one-loop penguin $b \rightarrow s, d$ transitions. Evidence for charmless $B$ decays was obtained in two body hadronic modes. The branching ratios of $B_{d, s}^{0}$ to $\pi^{+} \pi^{-}$or $K^{+} \pi^{-}$and $B_{u}^{-}$to $\rho^{0} \pi^{-}$or $K^{* 0} \pi^{-}$were found to be $\left(2.8_{-1.0}^{+1.5} \pm 0.2\right) \times 10^{-5}$ and $\left(1.7_{-0.8}^{+1.2} \pm 0.2\right) \times 10^{-4}$ respectively. The fraction of these decays with a charged kaon in the final state that is not from the spectator $s$ quark, was measured to be $0.58 \pm 0.18$. Upper limits were set at $90 \%$ confidence level on the branching ratios for three and four body charmless hadronic decays in the range of $(1-3) \times 10^{-4}$, for inclusive radiative $b \rightarrow s \gamma$ decays at $5.4 \times 10^{-4}$, for the exclusive radiative decays $B_{d}^{0} \rightarrow K^{*}(892)^{0} \gamma$ and $B_{s}^{0} \rightarrow \phi(1020) \gamma$ at $2.1 \times 10^{-4}$ and $7.0 \times 10^{-4}$ respectively, and for dineutrino decays, $b \rightarrow s \nu \bar{\nu}$, in the exclusive channels $B_{d}^{0} \rightarrow K^{*}(892)^{0} \nu \bar{\nu}$ and $B_{s}^{0} \rightarrow \phi(1020) \nu \bar{\nu}$ at $1.0 \times 10^{-3}$ and $5.4 \times 10^{-3}$ respectively. The limits on dineutrino decays constrain theories with a new $U(1)$ gauge boson coupling predominantly to the third family of fermions.
\end{abstract}


W.Adam ${ }^{50}$, T.Adye $^{37}$, E.Agasi ${ }^{31}$, I.Ajinenko ${ }^{42}$, R.Aleksan ${ }^{39}$, G.D.Alekseev ${ }^{16}$, R.Alemany ${ }^{49}$, P.P.Allport ${ }^{22}$, S.Almehed $^{24}$, U.Amaldi $^{9}$, S.Amato ${ }^{47}$, A.Andreazza ${ }^{28}$, M.L.Andrieux ${ }^{14}$, P.Antilogus ${ }^{9}$, W-D.Apel $^{17}$, Y.Arnoud ${ }^{39}$, B.Asman ${ }^{44}$, J-E.Augustin ${ }^{25}$, A.Augustinus ${ }^{9}$, P.Baillon ${ }^{9}$, P.Bambade ${ }^{19}$, F.Barao ${ }^{21}$, R.Barate ${ }^{14}$, M.Barbi ${ }^{47}$, D.Y.Bardin ${ }^{16}$, A.Baroncelli ${ }^{40}$, O.Barring ${ }^{24}$, J.A.Barrio ${ }^{26}$, W.Bartl ${ }^{50}$, M.J.Bates ${ }^{37}$, M.Battaglia ${ }^{15}$, M.Baubillier $^{23}$, J.Baudot ${ }^{39}$, K-H.Becks ${ }^{52}$, M.Begalli ${ }^{6}$, P.Beilliere ${ }^{8}$, Yu.Belokopytov ${ }^{9,53}$, A.C.Benvenuti ${ }^{5}$, M.Berggren $^{47}$, D.Bertini ${ }^{25}$, D.Bertrand ${ }^{2}$, F.Bianchi $^{45}$, M.Bigi $^{45}$, M.S.Bilenky ${ }^{16}$, P.Billoir ${ }^{23}$, D.Bloch $^{10}$, M.Blume $^{52}$, T.Bolognese ${ }^{39}$, M.Bonesini ${ }^{28}$, W.Bonivento ${ }^{28}$, P.S.L.Booth ${ }^{22}$, G.Borisov ${ }^{42}$, C.Bosio $^{40}$, O.Botner $^{48}$, E.Boudinov $^{31}$, B.Bouquet ${ }^{19}$, C.Bourdarios ${ }^{9}$, T.J.V.Bowcock ${ }^{22}$, M.Bozzo ${ }^{13}$, P.Branchini ${ }^{40}$, K.D.Brand ${ }^{36}$, T.Brenke $^{52}$, R.A.Brenner ${ }^{15}$, C.Bricman ${ }^{2}$, R.C.A.Brown ${ }^{9}$, P.Bruckman ${ }^{18}$, J-M.Brunet ${ }^{8}$, L.Bugge ${ }^{33}$, T.Buran ${ }^{33}$, T.Burgsmueller ${ }^{52}$, P.Buschmann ${ }^{52}$, A.Buys ${ }^{9}$, S.Cabrera ${ }^{49}$, M.Caccia ${ }^{28}$, M.Calvi ${ }^{28}$, A.J.Camacho Rozas ${ }^{41}$, T.Camporesi ${ }^{9}$, V.Canale ${ }^{38}$, M.Canepa $^{13}$, K.Cankocak ${ }^{44}$, F.Cao ${ }^{2}$, F.Carena ${ }^{9}$, L.Carroll ${ }^{22}$, C.Caso ${ }^{13}$, M.V.Castillo Gimenez ${ }^{49}$, A.Cattai ${ }^{9}$, F.R.Cavallo ${ }^{5}$, V.Chabaud ${ }^{9}$, Ph.Charpentier ${ }^{9}$, L.Chaussard ${ }^{25}$, J.Chauveau $^{23}$, P.Checchia ${ }^{36}$, G.A.Chelkov ${ }^{16}$, M.Chen ${ }^{2}$, R.Chierici ${ }^{45}$, P.Chliapnikov ${ }^{42}$, P.Chochula ${ }^{7}$, V.Chorowicz $^{9}$, J.Chudoba ${ }^{30}$, V.Cindro ${ }^{43}$, P.Collins ${ }^{9}$, J.L.Contreras ${ }^{19}$, R.Contri ${ }^{13}$, E.Cortina ${ }^{49}$, G.Cosme ${ }^{19}$, F.Cossutti $^{46}$, H.B.Crawley ${ }^{1}$, D.Crennell ${ }^{37}$, G.Crosetti ${ }^{13}$, J.Cuevas Maestro ${ }^{34}$, S.Czellar ${ }^{15}$, E.Dahl-Jensen ${ }^{29}$, J.Dahm $^{52}$, B.Dalmagne ${ }^{19}$, M.Dam ${ }^{29}$, G.Damgaard ${ }^{29}$, P.D.Dauncey ${ }^{37}$, M.Davenport ${ }^{9}$, W.Da Silva ${ }^{23}$, C.Defoix ${ }^{8}$, A.Deghorain $^{2}$, G.Della Ricca ${ }^{46}$, P.Delpierre ${ }^{27}$, N.Demaria ${ }^{35}$, A.De Angelis ${ }^{9}$, W.De Boer ${ }^{17}$, S.De Brabandere ${ }^{2}$, C.De Clercq ${ }^{2}$, C.De La Vaissiere ${ }^{23}$, B.De Lotto ${ }^{46}$, A.De Min ${ }^{36}$, L.De Paula ${ }^{47}$, C.De Saint-Jean ${ }^{39}$, H.Dijkstra ${ }^{9}$, L.Di Ciaccio ${ }^{38}$, F.Djama ${ }^{10}$, J.Dolbeau ${ }^{8}$, M.Donszelmann ${ }^{9}$, K.Doroba ${ }^{51}$, M.Dracos ${ }^{10}$, J.Drees ${ }^{52}$, K.-A.Drees ${ }^{52}$, M.Dris $^{32}$, J-D.Durand ${ }^{25}$, D.Edsall ${ }^{1}$, R.Ehret ${ }^{17}$, G.Eigen ${ }^{4}$, T.Ekelof ${ }^{48}$, G.Ekspong ${ }^{44}$, M.Elsing ${ }^{52}$, J-P.Engel ${ }^{10}$, B.Erzen ${ }^{43}$, M.Espirito Santo ${ }^{21}$, E.Falk ${ }^{24}$, D.Fassouliotis ${ }^{32}$, M.Feindt ${ }^{9}$, A.Fenyuk ${ }^{42}$, A.Ferrer ${ }^{49}$, S.Fichet ${ }^{23}$, T.A.Filippas ${ }^{32}$, A.Firestone ${ }^{1}$, P.-A.Fischer ${ }^{10}$, H.Foeth ${ }^{9}$, E.Fokitis ${ }^{32}$, F.Fontanelli ${ }^{13}$, F.Formenti ${ }^{9}$, B.Franek ${ }^{37}$, P.Frenkiel $^{8}$, D.C.Fries ${ }^{17}$, A.G.Frodesen ${ }^{4}$, R.Fruhwirth ${ }^{50}$, F.Fulda-Quenzer ${ }^{19}$, J.Fuster ${ }^{49}$, A.Galloni ${ }^{22}$,

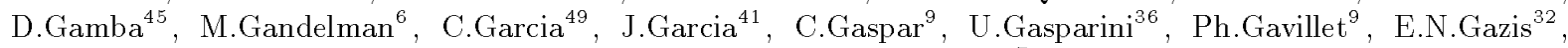
D.Gele ${ }^{10}$, J-P.Gerber ${ }^{10}$, M.Gibbs ${ }^{22}$, R.Gokieli ${ }^{51}$, B.Golob ${ }^{43}$, G.Gopal ${ }^{37}$, L.Gorn ${ }^{1}$, M.Gorski ${ }^{51}$, Yu.Gouz ${ }^{45,53}$, V.Gracco ${ }^{13}$, E.Graziani ${ }^{40}$, G.Grosdidier ${ }^{19}$, K.Grzelak ${ }^{51}$, S.Gumenyuk ${ }^{28,53}$, P.Gunnarsson ${ }^{44}$, M.Gunther ${ }^{48}$,

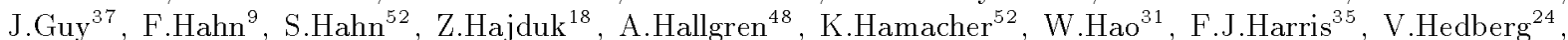
R.Henriques $^{21}$, J.J.Hernandez ${ }^{49}$, P.Herquet ${ }^{2}$, H.Herr ${ }^{9}$, T.L.Hessing ${ }^{35}$, E.Higon ${ }^{49}$, H.J.Hilke ${ }^{9}$, T.S.Hill ${ }^{1}$, S-O.Holmgren ${ }^{44}$, P.J.Holt ${ }^{35}$, D.Holthuizen ${ }^{31}$, S.Hoorelbeke ${ }^{2}$, M.Houlden ${ }^{22}$, J.Hrubec ${ }^{50}$, K.Huet ${ }^{2}$, K.Hultqvist ${ }^{44}$, J.N.Jackson $^{22}$, R.Jacobsson ${ }^{44}$, P.Jalocha ${ }^{18}$, R.Janik ${ }^{7}$, Ch.Jarlskog ${ }^{24}$, G.Jarlskog ${ }^{24}$, P.Jarry ${ }^{39}$, B.Jean-Marie ${ }^{19}$, E.K.Johansson ${ }^{44}$, L.Jonsson ${ }^{24}$, P.Jonsson ${ }^{24}$, C.Joram ${ }^{9}$, P.Juillot ${ }^{10}$, M.Kaiser ${ }^{17}$, F.Kapusta ${ }^{23}$, K.Karafasoulis ${ }^{11}$, M.Karlsson $^{44}$, E.Karvelas ${ }^{11}$, S.Katsanevas ${ }^{3}$, E.C.Katsoufis ${ }^{32}$, R.Keranen ${ }^{4}$, Yu.Khokhlov ${ }^{42}$, B.A.Khomenko ${ }^{16}$, N.N.Khovanski ${ }^{16}$, B.King ${ }^{22}$, N.J.Kjaer ${ }^{29}$, H.Klein ${ }^{9}$, A.Klovning ${ }^{4}$, P.Kluit ${ }^{31}$, B.Koene ${ }^{31}$, P.Kokkinias $^{11}$, M.Koratzinos $^{9}$, K.Korcyl ${ }^{18}$, C.Kourkoumelis ${ }^{3}$, O.Kouznetsov ${ }^{13,16}$, P.-H.Kramer ${ }^{52}$, M.Krammer ${ }^{50}$, C.Kreuter $^{17}$, I.Kronkvist $^{24}$, Z.Krumstein ${ }^{16}$, W.Krupinski ${ }^{18}$, P.Kubinec ${ }^{7}$, W.Kucewicz ${ }^{18}$, K.Kurvinen ${ }^{15}$, C.Lacasta ${ }^{49}$, I.Laktineh $^{25}$, S.Lamblot ${ }^{23}$, J.W.Lamsa ${ }^{1}$, L.Lanceri ${ }^{46}$, D.W.Lane ${ }^{1}$, P.Langefeld ${ }^{52}$, I.Last ${ }^{22}$, J-P.Laugier ${ }^{39}$, R.Lauhakangas $^{15}$, G.Leder ${ }^{50}$, F.Ledroit ${ }^{14}$, V.Lefebure ${ }^{2}$, C.K.Legan ${ }^{1}$, R.Leitner ${ }^{30}$, Y.Lemoigne ${ }^{39}$, J.Lemonne $^{2}$, G.Lenzen $^{52}$, V.Lepeltier ${ }^{19}$, T.Lesiak ${ }^{18}$, J.Libby ${ }^{35}$, D.Liko $^{50}$, R.Lindner ${ }^{52}$, A.Lipniacka $^{36}$, I.Lippi $^{36}$, B.Loerstad $^{24}$, J.G.Loken ${ }^{35}$, J.M.Lopez ${ }^{41}$, D.Loukas ${ }^{11}$, P.Lutz ${ }^{39}$, L.Lyons ${ }^{35}$, J.MacNaughton ${ }^{50}$, G.Maehlum ${ }^{17}$, A.Maio $^{21}$, V.Malychev ${ }^{16}$, J.Marco ${ }^{41}$, R.Marco ${ }^{41}$, B.Marechal ${ }^{47}$, M.Margoni ${ }^{36}$, J-C.Marin ${ }^{9}$, C.Mariotti ${ }^{40}$, A.Markou $^{11}$, T.Maron ${ }^{52}$, C.Martinez-Rivero ${ }^{41}$, F.Martinez-Vidal ${ }^{49}$, S.Marti i Garcia ${ }^{49}$, J.Masik ${ }^{30}$, F.Matorras $^{41}$, C.Matteuzzi ${ }^{9}$, G.Matthiae ${ }^{38}$, M.Mazzucato ${ }^{36}$, M.Mc Cubbin ${ }^{9}$, R.Mc Kay ${ }^{1}$, R.Mc Nulty ${ }^{22}$, J.Medbo $^{48}$, M.Merk ${ }^{31}$, C.Meroni ${ }^{28}$, S.Meyer ${ }^{17}$, W.T.Meyer ${ }^{1}$, A.Miagkov ${ }^{42}$, M.Michelotto ${ }^{36}$, E.Migliore ${ }^{45}$, L.Mirabito $^{25}$, W.A.Mitaroff ${ }^{50}$, U.Mjoernmark ${ }^{24}$, T.Moa ${ }^{44}$, R.Moeller ${ }^{29}$, K.Moenig ${ }^{9}$, M.R.Monge ${ }^{13}$, P.Morettini $^{13}$, H.Mueller ${ }^{17}$, L.M.Mundim ${ }^{6}$, W.J.Murray ${ }^{37}$, B.Muryn ${ }^{18}$, G.Myatt ${ }^{35}$, F.Naraghi ${ }^{14}$, F.L.Navarria ${ }^{5}$, S.Navas $^{49}$, K.Nawrocki ${ }^{51}$, P.Negri ${ }^{28}$, W.Neumann ${ }^{52}$, N.Neumeister ${ }^{50}$, R.Nicolaidou ${ }^{3}$, B.S.Nielsen ${ }^{29}$, M.Nieuwenhuizen $^{31}$, V.Nikolaenko ${ }^{10}, \quad$ P.Niss $^{44}, \quad$ A.Nomerotski $^{36}$, A.Normand ${ }^{35}$, M.Novak ${ }^{12}$, W.Oberschulte-Beckmann ${ }^{17}$, V.Obraztsov ${ }^{42}$, A.G.Olshevski ${ }^{16}$, A.Onofre ${ }^{21}$, R.Orava ${ }^{15}$, K.Osterberg ${ }^{15}$, A.Ouraou $^{39}$, P.Paganini ${ }^{19}$, M.Paganoni ${ }^{9}$, P.Pages ${ }^{10}$, H.Palka ${ }^{18}$, Th.D.Papadopoulou ${ }^{32}$, K.Papageorgiou ${ }^{11}{ }^{\text {, }}$ L.Pape $^{9}$, C.Parkes ${ }^{35}$, F.Parodi ${ }^{13}$, A.Passeri ${ }^{40}$, M.Pegoraro ${ }^{36}$, L.Peralta $^{21}$, H.Pernegger ${ }^{50}$, M.Pernicka ${ }^{50}$, A.Perrotta $^{5}$, C.Petridou ${ }^{46}$, A.Petrolini ${ }^{13}$, M.Petrovyck ${ }^{28,53}$, H.T.Phillips ${ }^{37}$, G.Piana ${ }^{13}$, F.Pierre ${ }^{39}$, M.Pimenta $^{21}$, M.Pindo $^{28}$, S.Plaszczynski ${ }^{19}$, O.Podobrin ${ }^{17}$, M.E.Pol ${ }^{6}$, G.Polok ${ }^{18}$, P.Poropat ${ }^{46}$, V.Pozdniakov ${ }^{16}$, M.Prest ${ }^{46}$, P.Privitera $^{38}$, N.Pukhaeva ${ }^{16}$, A.Pullia ${ }^{28}$, D.Radojicic ${ }^{35}$, S.Ragazzi ${ }^{28}$, H.Rahmani ${ }^{32}$, J.Rames ${ }^{12}$, P.N.Ratoff ${ }^{20}$, A.L.Read ${ }^{33}$, M.Reale ${ }^{52}$, P.Rebecchi ${ }^{19}$, N.G.Redaelli ${ }^{28}$, M.Regler ${ }^{50}$, D.Reid ${ }^{9}$, P.B.Renton ${ }^{35}$, L.K.Resvanis ${ }^{3}$, F.Richard ${ }^{19}$, J.Richardson ${ }^{22}$, J.Ridky ${ }^{12}$, G.Rinaudo ${ }^{45}$, I.Ripp ${ }^{39}$, A.Romero ${ }^{45}$, I.Roncagliolo ${ }^{13}$, P.Ronchese ${ }^{36}$, L.Roos $^{14}$, E.I.Rosenberg ${ }^{1}$, E.Rosso ${ }^{9}$, P.Roudeau ${ }^{19}$, T.Rovelli ${ }^{5}$, W.Ruckstuhl ${ }^{31}$, V.Ruhlmann-Kleider ${ }^{39}$, A.Ruiz ${ }^{41}$, K.Rybicki ${ }^{18}$, H.Saarikko ${ }^{15}$, Y.Sacquin ${ }^{39}$, A.Sadovsky ${ }^{16}$, O.Sahr ${ }^{14}$, G.Sajot ${ }^{14}$, J.Salt ${ }^{49}$, J.Sanchez ${ }^{26}$, M.Sannino $^{13}$, M.Schimmelpfennig ${ }^{17}$, H.Schneider ${ }^{17}$, U.Schwickerath ${ }^{17}$, M.A.E.Schyns ${ }^{52}$, G.Sciolla ${ }^{45}$, F.Scuri ${ }^{46}$, P.Seager $^{20}$, Y.Sedykh ${ }^{16}$, A.M.Segar ${ }^{35}$, A.Seitz $^{17}$, R.Sekulin ${ }^{37}$, R.C.Shellard ${ }^{6}$, I.Siccama ${ }^{31}$, P.Siegrist ${ }^{39}$, S.Simonetti $^{39}$, F.Simonetto ${ }^{36}$, A.N.Sisakian ${ }^{16}$, B.Sitar ${ }^{7}$, T.B.Skaali ${ }^{33}$, G.Smadja $^{25}$, N.Smirnov ${ }^{42}$, O.Smirnova $^{24}$, G.R.Smith $^{37}$, A.Sokolov ${ }^{42}$, O.Solovianov ${ }^{42}$, R.Sosnowski ${ }^{51}$, D.Souza-Santos ${ }^{6}$, T.Spassov ${ }^{21}$, E.Spiriti ${ }^{40}$, P.Sponholz ${ }^{52}$, S.Squarcia ${ }^{13}$, C.Stanescu ${ }^{40}$, S.Stapnes ${ }^{33}$, I.Stavitski ${ }^{36}$, K.Stevenson ${ }^{35}$, F.Stichelbaut $^{9}$, A.Stocchi $^{19}$, J.Strauss ${ }^{50}$, R.Strub ${ }^{10}$, B.Stugu ${ }^{4}$, M.Szczekowski ${ }^{51}$, M.Szeptycka ${ }^{51}$, T.Tabarelli ${ }^{28}$, J.P.Tavernet ${ }^{23}$, 
O.Tchikilev $^{42}$, J.Thomas ${ }^{35}$, A.Tilquin ${ }^{27}$, J.Timmermans ${ }^{31}$, L.G.Tkatchev ${ }^{16}$, T.Todorov ${ }^{10}$, S.Todorova $^{10}$, D.Z.Toet ${ }^{31}$, A.Tomaradze ${ }^{2}$, B.Tome ${ }^{21}$, A.Tonazzo ${ }^{28}$, L.Tortora ${ }^{40}$, G.Transtromer ${ }^{24}$, D.Treille ${ }^{9}$, W. Trischuk ${ }^{9}$, G.Tristram ${ }^{8}$, A.Trombini ${ }^{19}$, C.Troncon ${ }^{28}$, A.Tsirou ${ }^{9}$, M-L.Turluer ${ }^{39}$, I.A.Tyapkin ${ }^{16}$, M.Tyndel ${ }^{37}$, S.Tzamarias ${ }^{22}$, B.Ueberschaer ${ }^{52}$, O.Ullaland ${ }^{9}$, V.Uvarov ${ }^{42}$, G.Valenti ${ }^{5}$, E.Vallazza ${ }^{9}$, C.Vander Velde ${ }^{2}$, G.W.Van Apeldoorn ${ }^{31}$, P.Van Dam ${ }^{31}$, W.K.Van Doninck ${ }^{2}$, J.Van Eldik ${ }^{31}$, N.Vassilopoulos ${ }^{35}$, G.Vegni ${ }^{28}$, L.Ventura $^{36}$, W.Venus ${ }^{37}$, F.Verbeure ${ }^{2}$, M.Verlato ${ }^{36}$, L.S.Vertogradov ${ }^{16}$, D.Vilanova ${ }^{39}$, P.Vincent ${ }^{25}$, L.Vitale ${ }^{46}$, E.Vlasov $^{42}$, A.S.Vodopyanov ${ }^{16}$, V.Vrba ${ }^{12}$, H.Wahlen ${ }^{52}$, C.Walck $^{44}$, F.Waldner ${ }^{46}$, M.Weierstall ${ }^{52}$, P.Weilhammer $^{9}$, C.Weiser ${ }^{17}$, A.M.Wetherell ${ }^{9}$, D.Wicke ${ }^{52}$, J.H.Wickens ${ }^{2}$, M.Wielers ${ }^{17}$, G.R.Wilkinson ${ }^{35}$, W.S.C.Williams ${ }^{35}$, M.Winter ${ }^{10}$, M.Witek ${ }^{18}$, K.Woschnagg ${ }^{48}$, K.Yip ${ }^{35}$, O.Yushchenko ${ }^{42}$, F.Zach ${ }^{25}$, A.Zaitsev ${ }^{42}$, A.Zalewska $^{9}, \quad$ P.Zalewski ${ }^{51}, \quad$ D.Zavrtanik ${ }^{43}, \quad$ E.Zevgolatakos ${ }^{11}, \quad$ N.I.Zimin ${ }^{16}, \quad$ M.Zito $^{39}, \quad$ D.Zontar ${ }^{43}$, G.C.Zucchellit ${ }^{44}$, G.Zumerle ${ }^{36}$

\footnotetext{
${ }^{1}$ Ames Laboratory and Department of Physics, Iowa State University, Ames IA 50011, USA

${ }^{2}$ Physics Department, Univ. Instelling Antwerpen, Universiteitsplein 1, B-2610 Wilrijk, Belgium and IIHE, ULB-VUB, Pleinlaan 2, B-1050 Brussels, Belgium

and Faculté des Sciences, Univ. de l'Etat Mons, Av. Maistriau 19, B-7000 Mons, Belgium

${ }^{3}$ Physics Laboratory, University of Athens, Solonos Str. 104, GR-10680 Athens, Greece

${ }^{4}$ Department of Physics, University of Bergen, Allégaten 55, N-5007 Bergen, Norway

${ }^{5}$ Dipartimento di Fisica, Università di Bologna and INFN, Via Irnerio 46, I-40126 Bologna, Italy

${ }^{6}$ Centro Brasileiro de Pesquisas Físicas, rua Xavier Sigaud 150, RJ-22290 Rio de Janeiro, Brazil and Depto. de Física, Pont. Univ. Católica, C.P. 38071 RJ-22453 Rio de Janeiro, Brazil and Inst. de Física, Univ. Estadual do Rio de Janeiro, rua São Francisco Xavier 524, Rio de Janeiro, Brazil ${ }^{7}$ Comenius University, Faculty of Mathematics and Physics, Mlynska Dolina, SK-84215 Bratislava, Slovakia

${ }^{8}$ Collège de France, Lab. de Physique Corpusculaire, IN2P3-CNRS, F-75231 Paris Cedex 05, France

${ }^{9} \mathrm{CERN}, \mathrm{CH}-1211$ Geneva 23, Switzerland

${ }^{10}$ Centre de Recherche Nucléaire, IN2P3 - CNRS/ULP - BP20, F-67037 Strasbourg Cedex, France

${ }^{11}$ Institute of Nuclear Physics, N.C.S.R. Demokritos, P.O. Box 60228, GR-15310 Athens, Greece

${ }^{12}$ FZU, Inst. of Physics of the C.A.S. High Energy Physics Division, Na Slovance 2, 180 40, Praha 8, Czech Republic

${ }^{13}$ Dipartimento di Fisica, Università di Genova and INFN, Via Dodecaneso 33, I-16146 Genova, Italy

${ }^{14}$ Institut des Sciences Nucléaires, IN2P3-CNRS, Université de Grenoble 1, F-38026 Grenoble Cedex, France

${ }^{15}$ Research Institute for High Energy Physics, SEFT, P.O. Box 9, FIN-00014 Helsinki, Finland

${ }^{16}$ Joint Institute for Nuclear Research, Dubna, Head Post Office, P.O. Box 79, 101000 Moscow, Russian Federation

${ }^{17}$ Institut für Experimentelle Kernphysik, Universität Karlsruhe, Postfach 6980, D-76128 Karlsruhe, Germany

${ }^{18}$ Institute of Nuclear Physics and University of Mining and Metalurgy, Ul. Kawiory 26a, PL-30055 Krakow, Poland

${ }^{19}$ Université de Paris-Sud, Lab. de l'Accélérateur Linéaire, IN2P3-CNRS, Bât. 200, F-91405 Orsay Cedex, France

${ }^{20}$ School of Physics and Chemistry, University of Lancaster, Lancaster LA1 4YB, UK

${ }^{21}$ LIP, IST, FCUL - Av. Elias Garcia, 14-1 ${ }^{\circ}$, P-1000 Lisboa Codex, Portugal

${ }^{22}$ Department of Physics, University of Liverpool, P.O. Box 147, Liverpool L69 3BX, UK

${ }^{23}$ LPNHE, IN2P3-CNRS, Universités Paris VI et VII, Tour 33 (RdC), 4 place Jussieu, F-75252 Paris Cedex 05, France

${ }^{24}$ Department of Physics, University of Lund, Sölvegatan 14, S-22363 Lund, Sweden

${ }^{2.5}$ Université Claude Bernard de Lyon, IPNL, IN2P3-CNRS, F-69622 Villeurbanne Cedex, France

${ }^{26}$ Universidad Complutense, Avda. Complutense s/n, E-28040 Madrid, Spain

${ }^{27}$ Univ. d'Aix - Marseille II - CPP, IN2P3-CNRS, F-13288 Marseille Cedex 09, France

${ }^{28}$ Dipartimento di Fisica, Università di Milano and INFN, Via Celoria 16, I-20133 Milan, Italy

${ }^{29}$ Niels Bohr Institute, Blegdamsvej 17, DK-2100 Copenhagen 0, Denmark

${ }^{30} \mathrm{NC}$, Nuclear Centre of MFF, Charles University, Areal MFF, V Holesovickach 2, 180 00, Praha 8, Czech Republic

${ }^{31}$ NIKHEF-H, Postbus 41882, NL-1009 DB Amsterdam, The Netherlands

${ }^{32}$ National Technical University, Physics Department, Zografou Campus, GR-15773 Athens, Greece

${ }^{33}$ Physics Department, University of Oslo, Blindern, N-1000 Oslo 3, Norway

${ }^{34}$ Dpto. Fisica, Univ. Oviedo, C/P. Pérez Casas, S/N-33006 Oviedo, Spain

${ }^{35}$ Department of Physics, University of Oxford, Keble Road, Oxford OX1 3RH, UK

${ }^{36}$ Dipartimento di Fisica, Università di Padova and INFN, Via Marzolo 8, I-35131 Padua, Italy

${ }^{37}$ Rutherford Appleton Laboratory, Chilton, Didcot OX11 OQX, UK

${ }^{38}$ Dipartimento di Fisica, Università di Roma II and INFN, Tor Vergata, I-00173 Rome, Italy

${ }^{39}$ Centre d'Etudes de Saclay, DSM/DAPNIA, F-91191 Gif-sur-Yvette Cedex, France

${ }^{40}$ Istituto Superiore di Sanità, Ist. Naz. di Fisica Nucl. (INFN), Viale Regina Elena 299, I-00161 Rome, Italy

${ }^{41}$ Instituto de Fisica de Cantabria (CSIC-UC), Avda. los Castros, S/N-39006 Santander, Spain, (CICYT-AEN93-0832)

${ }^{42}$ Inst. for High Energy Physics, Serpukov P.O. Box 35, Protvino, (Moscow Region), Russian Federation

${ }^{43} \mathrm{~J}$. Stefan Institute and Department of Physics, University of Ljubljana, Jamova 39, SI-61000 Ljubljana, Slovenia

${ }^{44}$ Fysikum, Stockholm University, Box 6730, S-113 85 Stockholm, Sweden

${ }^{45}$ Dipartimento di Fisica Sperimentale, Università di Torino and INFN, Via P. Giuria 1, I-10125 Turin, Italy

${ }^{46}$ Dipartimento di Fisica, Università di Trieste and INFN, Via A. Valerio 2, I-34127 Trieste, Italy and Istituto di Fisica, Università di Udine, I-33100 Udine, Italy

${ }^{47}$ Univ. Federal do Rio de Janeiro, C.P. 68528 Cidade Univ., Ilha do Fundão BR-21945-970 Rio de Janeiro, Brazil

${ }^{48}$ Department of Radiation Sciences, University of Uppsala, P.O. Box 535, S-751 21 Uppsala, Sweden

${ }^{49}$ IFIC, Valencia-CSIC, and D.F.A.M.N., U. de Valencia, Avda. Dr. Moliner 50, E-46100 Burjassot (Valencia), Spain

${ }^{50}$ Institut für Hochenergiephysik, Österr. Akad. d. Wissensch., Nikolsdorfergasse 18, A-1050 Vienna, Austria

${ }^{51}$ Inst. Nuclear Studies and University of Warsaw, Ul. Hoza 69, PL-00681 Warsaw, Poland

${ }^{52}$ Fachbereich Physik, University of Wuppertal, Postfach 100 127, D-42097 Wuppertal 1, Germany

${ }^{53}$ On leave of absence from IHEP Serpukhov
} 


\section{Introduction}

This paper presents a study of three classes of rare charmless $b$ decays using the data collected in 1991-94 by the DELPHI experiment at LEP. The three classes of $b$ decays studied are the charmless decays in hadronic, radiative and dineutrino modes. These modes probe different decay processes contributing to the total charmless decay rate.

Charmless decays of the $b$ quark in the Standard Model are due both to tree level $b \rightarrow u$ diagrams (see Figs. $1 \mathrm{a}$ and $1 \mathrm{~b}$ ) and to one-loop penguin diagrams inducing $b \rightarrow d$ and $b \rightarrow s$ transitions (Fig. 1c). Non-spectator processes, such as exchange and annihilation diagrams, are expected to give only relatively minor contributions. Different decay modes can originate either from only one of these two classes of processes or from a combination of them. For example, semi-leptonic charmless decays are pure $b \rightarrow u$ transitions while decays involving $b \rightarrow s$ transitions are due to penguin loop diagrams, flavour changing neutral current processes being forbidden at tree level in the Standard Model. Charmless hadronic decays of $B$ particles receive contributions from both tree level $b \rightarrow u$ processes and penguin processes. Charmless radiative and dineutrino decays come purely from penguin diagrams (Figs. $1 \mathrm{~d}$ and $1 \mathrm{e}$ ). The total branching ratio into charmless final states is expected [1] to be a few percent in the Standard Model, with most of the individual modes contributing a few times $10^{-4}$ or less. The determination of their rates is a test of the loop structure of the Standard Model and can be used to constrain extensions involving new particles that can contribute to the internal lines in Figs. 1a-e.

With the data taken up to 1994 inclusive giving an integrated statistics of almost 3 million hadronic $Z^{0}$ decays per experiment, corresponding to about 1.3 million $b$ quark decays, experiments at LEP have reached a sensitivity at the level of the expected decay rates for several of these channels. The use of high resolution vertex detectors allows the secondary vertex topology of the decay of the long-flying $B$ hadron to be reconstructed, thus improving the signal to background ratio. In addition, the efficient hadron identification specific to the DELPHI detector is a powerful instrument for classifying the candidate events.

The paper is organised as follows. The components of the DELPHI detector most important for this study and the event samples used are first summarised briefly in section 2. Section 3 describes the analysis of exclusive hadronic charmless decays. These are important because the role of tree level and penguin contributions can be studied from the rates observed in different decay channels. The understanding of the penguin contribution to decays such as $B \rightarrow \pi \pi$ will be an essential ingredient in the studies and interpretation of $\mathrm{CP}$ asymmetries in $B$ decays at dedicated $B$ experiments [2]. Sections 4 and 5 describe the reconstruction techniques and the results obtained in the searches for $b \rightarrow s \gamma$ and $B \rightarrow K^{*} \nu \bar{\nu}$ decays. The conclusions are summarised in section 6 .

\section{The detector and generalities of the data analysis}

The DELPHI detector and its performance have been described elsewhere [3,4]. Of particular importance for this study are the identification of charged particles and photons and the precise track extrapolation to the neighbourhood of the $Z^{0}$ decay point, allowing the selection of $Z^{0} \rightarrow b \bar{b}$ decays and the reconstruction of their secondary vertices. 


\subsection{Charged particle identification}

Charged particle identification over a wide momentum range is an important feature of the DELPHI detector. Hadrons are identified by the combined use of the information derived from the specific ionisation in the Time Projection Chamber (TPC) and from the detection and measurement of rings of Cherenkov photons in the Ring Imaging Cherenkov detector (RICH). The absence of Cherenkov light in the RICH is also used ("veto mode").

The TPC provides up to 192 sampling points along the track giving a measurement of the rate of energy loss, $d E / d x$, to a precision of about $\pm 7 \%$ in hadronic events. This corresponds to separating kaons from pions by 1.5 standard deviations $(\sigma)$ for particles with momenta above $3.5 \mathrm{GeV} / c$.

The RICH gas radiator separates light particles $(e, \mu, \pi)$ from heavy ones $(K, p)$ above $3.5 \mathrm{GeV} / c$ and separates kaons from protons from 9 to about $20 \mathrm{GeV} / c[4,5]$. With the loose selection criteria used in this analysis, the efficiency for tagging a particle in this kinematic region is about 0.80 in the polar angle acceptance of the Barrel RICH, from $40^{\circ}$ to $140^{\circ}$, and is almost independent of the particle momentum.

For particles tagged as kaons, the rejection factor against misidentified pions is close to 5 using the $d E / d x$ information and from 8 to 5 , decreasing with increasing particle momentum, using the RICH detector. Identified charged particles were attributed their corresponding masses, unidentified ones were assumed to be pions.

\subsection{Photon identification}

Photons were detected by the barrel electromagnetic calorimeter, called the Highdensity Projection Chamber (HPC), located at a radius from the beam axis of between 208 and $260 \mathrm{~cm}$ and covering polar angles between $41^{\circ}$ and $139^{\circ}$. Its design provides full three-dimensional charge information of the electromagnetic showers. The spatial resolution is about $20 \mathrm{mrad}$ for the azimuthal angle while for the coordinate along the beam axis $(z)$ it is much more precise, varying between $1.3 \mathrm{~mm}$ and $3.1 \mathrm{~mm}$ depending on the polar angle. The energy resolution was measured to be $6 \%$ at $45 \mathrm{GeV}$ using Bhabha events.

Electromagnetic clusters not associated to a charged particle track were used to reconstruct photons and neutral pions. Single photons were distinguished from those from $\pi^{0}$ decays using different methods according to the energy. Low energy neutral pions were reconstructed by observing two clearly separated showers giving an invariant mass $m_{\gamma \gamma}$ compatible with this hypothesis. At energies above $5 \mathrm{GeV}$, the two showers are usually merged, and the three-dimensional reconstruction of showers in the fine grained HPC electromagnetic calorimeter was then used to separate photons from neutral pions. This was achieved by constrained fits to the shower shape using the profiles expected for the photon and $\pi^{0}$ hypotheses. For showers reconstructed in the HPC with energy larger than $5 \mathrm{GeV}$, and with the selection criteria used in this analysis, the efficiency for identifying photons correctly was estimated to be 0.6 , approximately independent of the energy, and the rejection against neutral pions varied between 3.5 and 2.0.

\subsection{Track extrapolations to the interaction region}

A three layer silicon vertex detector (VD) in the DELPHI tracking system ensures precise track reconstruction near the interaction region. In 1994 this detector was upgraded by replacing its innermost and outermost layers with double sided microstrip detector modules providing reconstruction of track points in space [6]. The innermost layer covers 
polar angles between $25^{\circ}$ and $155^{\circ}$. The accuracy of extrapolating tracks to the vertex was measured to be $\sqrt{\left(\left(65 /\left(p \sin ^{3 / 2} \theta\right)\right)^{2}+20^{2}\right)} \mu \mathrm{m}$ in the transverse plane $(R \phi)$ where $p$ is the particle momentum in $\mathrm{GeV} / \mathrm{c}$ and $\theta$ its polar angle. The precise track reconstruction obtained by the use of the vertex detector allows the use of secondary vertex reconstruction for the separation of $B$ decay products from primary hadronization particles. The reconstruction of these vertices is described below. In order to preserve an accurate track extrapolation to the vertex region, only tracks with at least one associated VD hit were used in the reconstruction of secondary vertices. This requirement was not applied for the reconstruction of $K_{s}^{0} \rightarrow \pi^{+} \pi^{-}$decaying outside the beam-pipe.

\subsection{Generalities of the data analysis}

Hadronic events were selected by the standard hadronic tag criteria [7]. This gave a total of $2.844 \times 10^{6} Z^{0}$ hadronic decay candidates from the 1991-1994 data set. The efficiency of the selection was determined to be $0.950 \pm 0.005$ on simulated data.

A $b$-tagging algorithm based on the significance, $d / \sigma$, where $d$ is the impact parameter and $\sigma$ its error, of the impact parameters of all the tracks in an event was applied to remove decays of the $Z^{0}$ into light quarks [8]. For each event, this algorithm gives the probability corresponding to the hypothesis that all the tracks originated at the $Z^{0}$ production point. Thus $Z^{0} \rightarrow u \bar{u}, d \bar{d}$ or $s \bar{s}$ decays give a flat probability distribution while $Z^{0} \rightarrow b \bar{b}$ decays give a pronounced spike near zero. Hadronic events were required to have a probability from the $b$-tagging algorithm smaller than 0.03 . This cut gives a selection efficiency of 0.85 for $Z^{0} \rightarrow b \bar{b}$ events and a purity of 0.67 .

Further enrichment in $b \bar{b}$ events was obtained by the specific requirements on vertex topology and kinematics in the different analyses, as described in the corresponding sections below. For the inclusive $b \rightarrow s \gamma$ analysis, only loose requirements on the secondary vertex were applied. For this reason the cut on the $b$-tagging probability was set at 0.01 , corresponding to an efficiency of 0.75 and a purity of 0.80 .

For each selected $Z^{0} \rightarrow b \vec{b}$ candidate, the primary vertex was fitted using a procedure that iteratively linked tracks to the beam-spot. Tracks not compatible with the beam-spot position were then removed. Secondary vertices were fitted using candidate secondary particles selected using kinematic and topological variables as described below for the different decay modes. A very loose $\chi^{2}$-probability cut at $10^{-5}$ was applied to reject combinations of tracks completely incompatible with the the hypothesis of originating from a common secondary vertex. The wrong association of fragmentation particles to the secondary vertex was strongly reduced by the requirements described later on the momenta, impact parameters and hadron identification of the tracks tested at this vertex. The invariant masses of the particles at the reconstructed vertices were determined with the track parameters recomputed at the fitted secondary vertex.

In reconstructing exclusive decay modes, further cuts on the vertex topology were applied to remove events with additional secondary tracks not used in the vertex reconstruction. A combination was rejected if at least one track, above $3 \mathrm{GeV} / \mathrm{c}$ and not used in the secondary vertex reconstruction, either missed the primary vertex by more than $2 \sigma$ or fitted the secondary vertex within $1 \sigma$, where $\pm \sigma$ is the $68 \%$ confidence level range after the convolution of the track extrapolation and the vertex reconstruction errors.

Charmless $b$ decays were considered to proceed via an intermediate resonance if the reconstructed mass of the relevant particles was within $2 \sigma$ of the resonance mass, where $\sigma$ is the $68 \%$ confidence level range after convoluting the natural width with the mass resolution. For $\phi \rightarrow K K$ decays, generally only one of the two kaons had to be identified, 
since the narrow mass cut around the $\phi$ mass already efficiently removed most of the combinatorial background.

The reconstruction and selection efficiencies of the different analyses were estimated using dedicated samples of fully simulated $Z^{0} \rightarrow b \bar{b}$ events, where one of the $b$ hadrons was forced to decay into the final states of interest. Using the decay distance and impact parameters reconstructed in space increased these efficiencies by between $15 \%$ and $25 \%$ in the 1994 data set compared with the data taken earlier when the VD was equipped with only $R \phi$ read-out.

The backgrounds for the different decay modes were evaluated using 5.2 million $Z^{0} \rightarrow$ $q \bar{q}$ and 1.6 million $Z^{0} \rightarrow b \bar{b}$ generated events processed with the full detector simulation. These simulation statistics are equivalent to about four times the real data set. The configuration of the DELPHI detector changed over the years, in particular for the VD. Simulation samples corresponding to the configurations in the different years were used in proportions close to those of the real data set.

To extract the results, the numbers of candidate events selected in the real data were compared with the numbers expected from the simulation of the background. In each case, the probability for the background level to fluctuate to a number of events equal to or larger than that observed in the real data was computed taking into account the uncertainty in the background estimate. Only decay modes for which this probability was less than $10^{-3}$ were considered for deriving branching ratios.

In all other cases, only upper limits at $90 \%$ confidence level are quoted. These upper limits were obtained from the number of events observed in the data taking into account the estimated background. If the number of background-subtracted events in the data was negative, though compatible with zero, the upper limit was conservatively computed assuming it to be zero. The effects of the systematic uncertainties on the signal efficiency and on the background estimate were included in the computation of the branching ratio upper limits.

Since the $B$ mass resolution in several exclusive decay channels receiving contributions from both the $B_{d}^{0}$ and the $B_{s}^{0}$ mesons was not high enough to separate the two $B$ meson species, results for these channels were computed for the sum of the $B_{d}^{0}$ and the $B_{s}^{0}$ decays, i.e. from the total number of candidate events and the total number of $B_{d}^{0}$ and $B_{s}^{0}$ mesons. Suppressed decay modes were not taken into account.

\section{Hadronic charmless decays}

In the Standard Model, hadronic charmless $B$ decays originate both from tree level spectator $b \rightarrow u$ transitions (Figs. 1a, 1b), and from the one-loop penguin process in which the $b$ decays to an $s$ quark via a loop including a virtual $W^{-}$boson and a virtual $t$ quark (Fig. 1c) and at least one gluon is emitted, giving the $b \rightarrow s g, s g g$ and $b \rightarrow s q \bar{q}$ processes [1].

Final states with a kaon are due either to $b \rightarrow s$ transitions or to Cabibbo suppressed $b \rightarrow u+s \bar{u}$ decays where the $K$ meson originates from the $W$ boson. Those with only pions are due mainly to $b \rightarrow u$ tree level diagrams. For example, exclusive channels such as $B^{0} \rightarrow \pi^{+} \pi^{-}$are mostly $b \rightarrow u$ decays with a possible contribution from suppressed $b \rightarrow d$ loop transitions. The decay $B^{0} \rightarrow K^{+} \pi^{-}$is due to a mixture of $b \rightarrow s$ and $b \rightarrow u+s \bar{u}$ decays. Finally decays with a neutral kaon, such as $B^{-} \rightarrow K^{* 0} \pi^{-}$, do not receive tree level contributions and are pure penguin processes.

Signals for hadronic charmless $b$ quark decays were first reported by CLEO [9] in the sum of the $B \rightarrow \pi \pi$ and $K \pi$ decay modes. At LEP, analyses have been performed by 
all four collaborations [10-13]. The analysis of the data collected by DELPHI from 1991 to 1993 provided an excess of events in two-body hadronic channels not compatible with charmed decay modes. This was interpreted as evidence for charmless decays of $B$ mesons [11]. Adding the 1994 data sample has almost doubled the statistics available compared with the previous study. Furthermore, the upgraded DELPHI microvertex detector (VD) has improved the efficiency for tagging $b \bar{b}$ events, reconstructing $B$ decay vertices, and separating particles from the $B$ decays from those due to fragmentation.

The particle identification capabilities of the DELPHI Ring Imaging Cherenkov detector allow the separation of channels with charged kaons from multi-pion final states. The determination of the fraction of candidate charmless events containing a kaon in the final state probes the relative contributions of the tree level and penguin loop processes to the rate of rare hadronic charmless modes. In addition, rare decays of the $\Lambda_{b}^{0}$ beauty baryon can be tagged due to the presence of an identified proton in the final state.

\subsection{Event reconstruction}

Events fulfilling the hadronic and $b$-tagging criteria were divided into two hemispheres. For each hemisphere the leading charged particle was used to start the secondary vertex reconstruction. Other charged particles were iteratively tested to form a common $n$-prong detached vertex with this leading particle.

Kinematic cuts were applied to exploit the hard $b$ fragmentation. The total energy of the $B$ candidate $E_{B}$ was required to be above $20 \mathrm{GeV}$ and below the beam energy. For two (greater than two) prong topologies, the momentum of the leading particle had to be larger than $10(8) \mathrm{GeV} / c$ and that of the other secondary particles larger than 1.0 (0.8) $\mathrm{GeV} / c$.

The combinatorial background was suppressed by requiring the candidate secondary vertex to be separated by more than $2.5 \sigma$ from the reconstructed primary vertex and the decay distance to be smaller than $2.0 \mathrm{~cm}$. Partially reconstructed $B$ decays give a background that falls steeply in the invariant mass distribution up to about $5.0 \mathrm{GeV} / \mathrm{c}^{2}$ for $B$ mesons. Above this value, a rather flat tail extends to higher masses. While partially reconstructed decays of beauty baryons also contribute below the $\Lambda_{b}$ mass value, this tail is due mainly to tracks from the primary vertex being incorrectly assigned to the secondary vertex. This was suppressed by requiring every track used in the secondary vertex reconstruction to have an impact parameter with respect to the primary vertex larger than 1.5 times its associated error. In the 1994 data both the $R \phi$ and the $z$ projections of the impact parameters were tested and at least one of the two was required to fulfil this cut. For earlier data only the $R \phi$ projection, where the track extrapolation accuracy was determined by the VD, was used. In two prong topologies this cut was not applied to the leading particle because simulation showed that, in low multiplicity $B$ decays, the leading particle is a $B$ decay product in more than $98 \%$ of the cases.

The following decay modes and their corresponding charge conjugates were investigated:

- Two-body decays:

$-B_{d}^{0} \rightarrow \pi^{+} \pi^{-}$

$-B_{u}^{-} \rightarrow \rho(770)^{0} \pi^{-}, \quad \rho(770)^{0} \rightarrow \pi^{+} \pi^{-}$

$-B_{d, s}^{0} \rightarrow K^{+} \pi^{-}$

$-\Lambda_{b}^{0} \rightarrow p K^{-}$

$-B_{u}^{-} \rightarrow K^{-} \rho(770)^{0}, \quad \rho(770)^{0} \rightarrow \pi^{+} \pi^{-}$

$-B_{d, s}^{0} \rightarrow K^{+} a_{1}(1270)^{-}, \quad a_{1}(1270)^{-} \rightarrow \pi^{-} \pi^{+} \pi^{-}$ 
$-B_{u}^{-} \rightarrow \bar{K}^{*}(892)^{0} \pi^{-}, \quad \bar{K}^{*}(892)^{0} \rightarrow K^{-} \pi^{+}$

$-B_{d, s}^{0} \rightarrow K^{+} K^{-}$

$-B_{u}^{-} \rightarrow \phi(1020) K^{-}, \quad \phi(1020) \rightarrow K^{+} K^{-}$

- Three-body decays:

$-B_{u}^{-} \rightarrow \pi^{-} \pi^{+} \pi^{-}$

$-B_{u}^{-} \rightarrow K^{-} \pi^{+} \pi^{-}$

$-B_{u}^{-} \rightarrow K^{-} K^{+} K^{-}$

- Four body decays:

$-B_{d}^{0} \rightarrow \pi^{+} \pi^{-} \pi^{+} \pi^{-}$

$-B_{d, s}^{0} \rightarrow K^{+} \pi^{-} \pi^{+} \pi^{-}$

In two-body decay channels involving a vector and a pseudo-scalar meson, such as $B \rightarrow$ $K^{*} \pi$ and $\rho \pi$, the vector meson is fully polarized. The distribution of the decay helicity angle $\theta^{*}$ in the vector meson rest frame is proportional to $\cos ^{2} \theta^{*}$ while the background is more isotropic. Therefore in these channels $\left|\cos \theta^{*}\right|$ was required to be larger than 0.5 .

In multi-prong final states, $B$ decays into fully reconstructed intermediate states containing hidden or open charm were removed by requiring that no pair of particles had an invariant mass close to either the $J / \psi$ or the $\psi(2 S)$ mass if given electron or muon masses, or close to the $D^{0}$ if assigned either $K \pi$ or $\pi \pi$ masses. The widths of the invariant mass intervals around the charm states corresponded to a $3 \sigma$ cut, using the measured mass. For four-prong vertices which had masses consistent with $B$ decay (see below), all combinations of three particles had to be incompatible both with the $D^{-}$mass when assigned $K^{+} \pi^{-} \pi^{-}$or $\pi^{+} \pi^{-} \pi^{-}$masses and with the $D_{s}$ when assigned $K^{+} K^{-} \pi^{-}$masses.

The mass resolutions were found to be $85 \mathrm{MeV} / \mathrm{c}^{2}, 60 \mathrm{MeV} / \mathrm{c}^{2}$ and $45 \mathrm{MeV} / \mathrm{c}^{2}$ for two, three and four prong decays respectively. The $B_{d, u}\left(B_{s}\right)$ candidates were accepted in the invariant mass region defined by the intervals $5.15-5.55 \mathrm{GeV} / c^{2}\left(5.25-5.65 \mathrm{GeV} / c^{2}\right)$ and $5.20-5.50 \mathrm{GeV} / c^{2}\left(5.30-5.60 \mathrm{GeV} / c^{2}\right)$ for two and more than two prongs respectively. $\Lambda_{b}^{0}$ candidates were accepted in the region between $5.45 \mathrm{GeV} / \mathrm{c}^{2}$ and $5.9 \mathrm{GeV} / \mathrm{c}^{2}$. Since the main source of background is partially reconstructed $B$ decays, the background is higher for masses below the $B_{d, u}$ meson mass. For this reason the signal mass regions were chosen to be asymmetric around the mass values of the different $b$ species.

\subsection{Results}

The characteristics of the candidate $B$ hadron decays into two, three and four bodies are given in Tables 1,2 and 3.

In two-body modes, eight candidates to be charmless decays of $B$ mesons were reconstructed in the full 1991 - 1994 statistics: two in the $\pi^{+} \pi^{-}$channel, three in $K^{+} \pi^{-}$, one in $\rho^{0} \pi^{-}$and two in $K^{* 0} \pi^{-}$(see Fig. 2). One of the two candidates classified as a $B \rightarrow \pi^{+} \pi^{-}$decay is ambiguous with the $K^{+} \pi^{-}$hypothesis, since the lower momentum hadron has no particle identification information. Changing from the $\pi \pi$ to the $K \pi$ mass assignment moves its total mass from $5.18 \mathrm{GeV} / \mathrm{c}^{2}$ to $5.32 \mathrm{GeV} / \mathrm{c}^{2}$, which is also inside the signal mass region. No candidates for charmless $\Lambda_{b}$ decays were found.

The background was estimated by studying the rejection factors on simulated $q \bar{q}$ events for independent sets of the cuts applied in the analysis. The secondary vertex selection criteria were relaxed for the two-prong sample. The effects of the intermediate mass and helicity angle constraints were also studied in the multi-prong sample. In addition the background suppression coming from kaon or proton identification was measured for all the channels. The rejection factors obtained from simulated events were compared with 
those obtained for real data and found to be consistent. This study gave $0.68 \pm 0.15$ background events.

The background estimate was checked with the number of simulated events fulfilling all the selection cuts in the mass interval from $5.1 \mathrm{GeV} / \mathrm{c}^{2}$ to $6.0 \mathrm{GeV} / \mathrm{c}^{2}$, normalised to the signal region and to the equivalent data statistics. The result was $0.75 \pm 0.25$ events, in agreement with the value estimated using the rejection factors.

Thus in two body hadronic modes, eight charmless decay candidates were selected in real data with an estimated background of $0.68 \pm 0.15$ events. The probability that all the events seen are due to a fluctuation of this background is $10^{-6}$. This result confirms with higher significance the evidence for charmless decays of $B$ mesons in two-body final states already reported by DELPHI based on the 1991-1993 data [11].

In three and four-body decay modes, five and three candidate decays were found respectively. These numbers are consistent with the respective estimated backgrounds of $3.5 \pm 1.0$ and $5.3 \pm 1.2$ events from other processes.

\subsection{Discussion}

The increased statistics allow the extraction of quantitative results for the rates observed in two body charmless decay channels, in particular the branching ratios for channels with a significant excess of events in the data, and the extraction of the fraction of events with a kaon in the final state that does not originate from a spectator $s$ quark.

\subsubsection{Branching ratios}

In the $\pi^{+} \pi^{-}$and $K^{+} \pi^{-}$modes, five events were observed with an estimated background of $0.15 \pm 0.05$ events. These numbers correspond to a probability of originating from a background fluctuation of $5 \times 10^{-5}$. The efficiency for reconstructing either $B^{0} \rightarrow \pi^{+} \pi^{-}$ or $B^{0} \rightarrow K^{+} \pi^{-}$decays without distinguishing between the two final states was evaluated to be $0.25 \pm 0.01$ using simulated data. The uncertainty on the reconstruction efficiency was taken into account as a systematic error. Interpreting all the five observed events as signal, the corresponding branching ratio is:

$$
\operatorname{BR}\left(B_{d, s}^{0} \rightarrow \pi^{+} \pi^{-}, K^{+} \pi^{-}\right)=\left(2.8_{-1.0}^{+1.5} \text { (stat.) } \pm 0.2 \text { (syst.) }\right) \times 10^{-5}
$$

This result agrees with the measurement reported by the CLEO Collaboration of $(2.4 \pm 0.8) \times 10^{-5}[9]$.

The exclusive $K^{+} \pi^{-}$channel has three events with an estimated background of $0.06 \pm$ 0.03. This corresponds to a probability of the events being a fluctuation of the background of $10^{-4}$. The three events give a branching ratio for this exclusive channel of:

$$
\operatorname{BR}\left(B_{d, s}^{0} \rightarrow K^{+} \pi^{-}\right)=\left(2.4_{-1.1}^{+1.7} \text { (stat.) } \pm 0.2 \text { (syst.) }\right) \times 10^{-5}
$$

For the decay into three-prong final states, the combination of the $\rho \pi$ and $K^{*} \pi$ channels gave three events in real data with an expected background of $0.15 \pm 0.04$ events. This excess is also significant, while for either of the individual channels the probability of a background fluctuation is above $10^{-3}$. Again considering the three candidates as signal events, the value for the combined branching ratio is:

$$
\left.\operatorname{BR}\left(B_{u}^{-} \rightarrow \rho \pi, K^{*} \pi\right)=\left(1.7_{-0.8}^{+1.2} \text { (stat.) } \pm 0.2 \text { (syst. }\right)\right) \times 10^{-4}
$$

In three and four-body modes, where no excess of events was observed, the upper limits set by this analysis are within a factor two of the expectations for these channels. The results are summarised in Tables 4,5 and 6 for two, three and four-body decays respectively. 


\subsubsection{Kaon fraction}

The fraction of candidates in two body modes containing a kaon in the final state was also measured. As can be seen by comparing Figs. 1a and 1b with Fig. 1c, this fraction is sensitive to the relative importance of the tree level and penguin contributions to the decay.

An unbinned maximum likelihood fit was performed using the reconstructed invariant mass $m_{B}$, the average Cherenkov angle $\bar{\theta}_{c}$ and the specific ionization $d E / d x$ as inputs. All the events with at least one of the $\pi$ or $K$ mass assignments, independently of the hadron tagging, giving an invariant mass in the $B$ mass signal region were used in the fit. ${ }^{\dagger}$ The efficiency for a signal event to be accepted in the fit is $0.26 \pm 0.01$. The fraction $\left.F\left[K \pi,(K \pi)_{K^{*}} \pi\right)\right]=N\left[K \pi,(K \pi)_{K^{*}} \pi\right] /\left\{N\left[\pi \pi,(\pi \pi)_{\rho} \pi\right]+N\left[K \pi,(K \pi)_{K^{*}} \pi\right]\right\}$ was left free in the fit. The result was $0.68_{-0.16}^{+0.13}$.

Decays of $B_{u, d}$ mesons may produce a charged kaon from either the $b \rightarrow s$ or the Cabibbo suppressed $b \rightarrow u s \bar{u}$ decay. In the case of the strange beauty meson $B_{s}^{0}$, the charged kaon can originate from the spectator $s$ quark also in the Cabibbo allowed tree level $b \rightarrow u d \bar{u}$ decay. This contribution was taken into account in a second fit made to estimate the fraction of $b \rightarrow K$ decays for which a charged kaon in the final state is not due to a spectator $s$ quark. The fraction of events containing a kaon and the fraction of $B_{s}^{0}$ candidates were fitted at the same time.

The result of the fit was that the fraction of candidate charmless hadronic $B$ decays for which a charged kaon in the final state is not due to a spectator $s$ quark is:

$$
F(b \rightarrow K)=0.58 \pm 0.18 \text {. }
$$

The fit gave $1.3_{-1.3}^{+1.5}$ for the number of $B_{s}^{0}$ candidates in the sample, to be compared with 1.5 expected assuming the $B_{s} / B_{d}$ production ratio $f_{s} / f_{d}=0.30$. Restricting the analysis to the five candidates in the two prong modes $B^{0} \rightarrow \pi^{+} \pi^{-}$and $K^{+} \pi^{-}$, the corresponding fraction $F\left(B_{d}^{0} \rightarrow K^{+} \pi^{-}\right)=N\left(B_{d}^{0} \rightarrow K^{+} \pi^{-}\right) /\left[N\left(B^{0} \rightarrow \pi^{+} \pi^{-}\right)+N\left(B^{0} \rightarrow K^{+} \pi^{-}\right)\right]$is determined to be $0.52 \pm 0.21$. These numbers are consistent with those expected if the penguin $b \rightarrow s$ and tree level $b \rightarrow u$ transitions contribute equally to the hadronic charmless $b$ decays.

\section{Radiative charmless decays}

The radiative decay $b \rightarrow s \gamma$ has recently been the focus of much interest from both theory and experiments. This decay (see Fig. 1d) proceeds through a one-loop penguin process, similar to the one contributing to the hadronic modes, in which a photon is radiated from either the $W^{-}$or the quark line. The rate for this decay has been computed in the Standard Model. Including part of the next-to-leading order QCD corrections gives $B R(b \rightarrow s \gamma)=(1.9 \pm 0.5) \times 10^{-4}[19]$. Additional contributions can come from new particles in the loop, such as charged Higgs bosons or supersymmetric particles. These contributions can either increase or decrease the $b \rightarrow s \gamma$ decay rate compared with the Standard Model expectation [20,21].

Evidence for $b \rightarrow s \gamma$ decays has been reported by CLEO both in the exclusive channel $B \rightarrow K^{*}(892) \gamma[22]$ and in the inclusive mode [23]. The inclusive rate observed corresponds to a $B R(b \rightarrow s \gamma)$ of $(2.32 \pm 0.67) \times 10^{-4}$, in good agreement with the Standard Model expectation. Searches for radiative charmless decays have already been performed at LEP on limited statistics by the DELPHI [24] and L3 [25] experiments.

\footnotetext{
${ }^{\dagger}$ No events other than the eight candidates were accepted with this looser requirement.
} 


\subsection{Event reconstruction}

Radiative charmless decays have been searched for in both the inclusive $b \rightarrow s \gamma$ and the exclusive $B_{d}^{0} \rightarrow K^{* 0} \gamma$ channels. The $b \rightarrow s \gamma$ analysis uses an inclusive algorithm to reconstruct the hadronic system accompanying the photon in the decay of the $B$ hadron. This method minimizes the dependence of the result on the size of the contribution from the individual exclusive decay channels.

In the search for fully reconstructed exclusive decays, two different procedures were followed and the results were combined. In the first one, the events reconstructed using the inclusive algorithm were tested against the hypothesis of being fully reconstructed decays. The second analysis used a dedicated exclusive reconstruction procedure similar to the one used for the study of the charmless hadronic decays.

\subsubsection{Inclusive search}

Hadronic events were selected that satisfied the $b$-tagging criteria and had a neutral electromagnetic shower reconstructed in the HPC calorimeter with energy larger than $6 \mathrm{GeV}$ and an energy component transverse to the jet axis $E_{t}$ above $0.7 \mathrm{GeV}$. Neutral pions were rejected by discarding all photons giving an invariant mass smaller than $0.25 \mathrm{GeV} / \mathrm{c}^{2}$ when paired with another photon, or by the shape of the electromagnetic showers as described in section 2. The accompanying hadronic system was reconstructed using an inclusive procedure. Candidate secondary particles were selected among those contained in a cone of $0.7 \mathrm{rad}$ around the photon direction and with momentum larger than $1 \mathrm{GeV} / \mathrm{c}$. Pairs of charged particle tracks, sorted in decreasing order of significance of their impact parameters with respect to the primary vertex, were iteratively tested for forming a common detached vertex. Vertices having low probability or a flight distance smaller than 0.5 times the associated error were discarded. When a vertex was accepted, other charged particle tracks and reconstructed neutral pions and $K_{s}^{0}$, sorted in decreasing order of rapidity, were tested for inclusion. Charged particles compatible with the vertex position and neutrals with rapidity larger than 1.5 were added, provided the total invariant mass of the hadronic system and the photon did not exceed $6 \mathrm{GeV} / \mathrm{c}^{2}$. No more than one neutral pion and one neutral kaon were associated to a vertex. Events with at least two secondary particles selected in addition to the photon, a minimum mass of the hadronic system larger than the $K^{0}$ mass and a minimum total energy of $15 \mathrm{GeV}$, of which at least $5 \mathrm{GeV}$ came from hadronic particles, were accepted. A study of simulated $b \rightarrow s \gamma$ events showed that on average $70 \%$ of the selected particles were genuine $B$ decay products.

Candidate events were selected by further requiring the total invariant mass to be between $3.25 \mathrm{GeV} / \mathrm{c}^{2}$ and $6.0 \mathrm{GeV} / \mathrm{c}^{2}$ and the mass of the selected hadronic system to be below $1.6 \mathrm{GeV} / c^{2}$. The dominant background is due to $b \rightarrow c$ decays with a misidentified $\pi^{0}$ or an energetic photon. The cut on the mass of the hadronic system removes a large fraction of this background, while it retains most of the signal, since the predicted invariant mass distribution of the hadronic system in $b \rightarrow s \gamma$ decays peaks below $1.5 \mathrm{GeV} / \mathrm{c}^{2}[26]$.

The energy of the $B$ hadron was estimated by scaling the sum of the energies of the selected particles and of the photon by the ratio of the total visible mass to the $B$ meson mass. This procedure resulted in an energy resolution of about $7 \%$ for the selected signal events. Events with a scaled energy above $20 \mathrm{GeV}$ were retained and the reconstructed photon was boosted into the $B$ rest frame. Signal $b \rightarrow s \gamma$ events give an almost mono-energetic photon in this rest frame with energy $E^{*}$ close to the kinematic limit of $\left(m_{b}^{2}-m_{s}^{2}\right) /\left(2 m_{b}\right)=2.4 \mathrm{GeV}$. The accuracy of the determination of the photon 
energy, $E^{*}$, in the $B$ rest frame was studied using simulated $b \rightarrow s \gamma$ events. The resolution function was extracted from the difference between the reconstructed and generated $E^{*}$ values after all the selection cuts. This resolution function is well described by the sum of two Gaussian distributions having $\sigma$ widths of $40 \mathrm{MeV}$ and $110 \mathrm{MeV}$ with the narrower of the two distributions containing $40 \%$ of the decays.

The detailed shape of the photon spectrum was obtained from a fully inclusive spectator model that uses a $B$ meson wave function model and includes gluon bremstrahlung and higher order radiative effects $[26,27]$. The input parameter values chosen were $m_{B}=$ $5.279 \mathrm{GeV} / \mathrm{c}^{2}, m_{\text {top }}=180 \mathrm{GeV} / \mathrm{c}^{2}$ and $p_{F}=0.27 \mathrm{GeV} / \mathrm{c}$, where $p_{F}$ is the Fermi motion of the $b$ quark in the hadron, while the spectator quark mass $m_{q}$ was set to zero. In the simulation of the signal events, the branching ratios for the different $K^{*}$ states were taken from the same model [28] except for the $K^{*}(892)$ for which the central value of the CLEO measurement [23] of $B R\left(B \rightarrow K^{*}(892) \gamma\right) / B R(B \rightarrow s \gamma)=0.19$ was used. In addition, $27 \%$ of the radiative decays were allowed to produce multi-body final states for which the JETSET fragmentation scheme was used [29]. Using this simulation, the efficiency for the above reconstruction procedure was found to be $0.043 \pm 0.002$. For comparison, using this efficiency, the central value measured by the CLEO collaboration for $B R(b \rightarrow s \gamma)$ corresponds to about 11 signal events reconstructed in the DELPHI data.

\subsubsection{Exclusive search}

Exclusive radiative $b$ decays were searched for in the channels:

- $B_{d}^{0} \rightarrow K^{*}(892)^{0} \gamma, K^{*}(892)^{0} \rightarrow K^{-} \pi^{+}$

- $B_{s}^{0} \rightarrow \phi(1020) \gamma, \quad \phi(1020) \rightarrow K^{+} K^{-}$.

Two reconstruction algorithms were used.

In the first one, further selections were made from the events reconstructed in the inclusive $b \rightarrow s \gamma$ analysis by taking those for which the hadronic system was consistent with $K^{*}(892)^{0} \rightarrow K^{-} \pi^{+}\left(\phi \rightarrow K^{+} K^{-}\right)$and the $K \pi \gamma(K K \gamma)$ mass corresponded to the $B$ meson mass. Candidate events were selected in the mass region $4.9 \mathrm{GeV} / \mathrm{c}^{2}<\mathrm{M}(K \pi \gamma)$ $<5.7 \mathrm{GeV} / \mathrm{c}^{2}$ and $5.0 \mathrm{GeV} / \mathrm{c}^{2}<\mathrm{M}(K K \gamma)<5.8 \mathrm{GeV} / \mathrm{c}^{2}$.

The second algorithm looked for displaced secondary $K \pi(K K)$ vertices associated with an energetic photon. This procedure profited from the clear signature of a charged kaon tagged by either the RICH detector or the $\mathrm{dE} / \mathrm{dx}$ of the TPC emitted in a cone of $0.7 \mathrm{rad}$ around the photon direction. The candidate kaon was tested to form a displaced secondary vertex with each of the other tracks having momentum above $1.0 \mathrm{GeV} / \mathrm{c}$. Pairs having an invariant mass compatible with that of the $K^{*}(892)^{0}$ or $\phi$ meson were associated with the photon and the total mass of the corresponding $K \pi \gamma$ or $K K \gamma$ system was computed. The selection criteria were similar to those of the $b \rightarrow s \gamma$ analysis for the photon and to those of the hadronic charmless decays for the charged particles. The photon was required to have more than $6 \mathrm{GeV}$, the momentum of the tagged kaon had to be above $3.5 \mathrm{GeV} / \mathrm{c}$ and the sum of the photon and $K^{*}$ energies had to exceed $25 \mathrm{GeV}$.

The $B$ mass resolution obtained from simulated signal events was $0.25 \mathrm{GeV} / c^{2}$ for both selection algorithms. The efficiency was computed with fully simulated $B^{0} \rightarrow K^{*}(\phi) \gamma$ events. Taking into account the fraction of signal events tagged by both procedures, the total efficiency is $0.076 \pm 0.008(0.075 \pm 0.010)$. Using this efficiency, the central value of the CLEO measurement of $B R\left(B \rightarrow K^{*} \gamma\right)=(4.5 \pm 1.7) \times 10^{-5}$ [22] corresponds to 2 fully reconstructed decays in the DELPHI data sample. 


\subsection{Results}

\subsubsection{Inclusive search}

The fraction of inclusive $b \rightarrow s \gamma$ candidates selected in real data by these cuts was extracted by a fit to the shape of the spectrum of the boosted photon energy. The signal was described by the predicted photon spectrum described above smeared with the resolution function obtained using simulated signal events. The background was modelled using fully simulated hadronic events not containing $b \rightarrow s \gamma$ decays and fulfilling the same cuts as the real data.

No excess of events in real data was observed and the fit gave $1 \pm 12$ signal events or $\operatorname{BR}(b \rightarrow s \gamma)=(0.2 \pm 2.5) \times 10^{-4}$ (Fig. 3). This corresponds to an upper limit of 20 events at $90 \%$ confidence level or $B R(b \rightarrow s \gamma)$ of $4.4 \times 10^{-4}$. The ratio of events selected in real and simulated data in the full spectrum of $E^{*}$ was $1.03 \pm 0.06$. In the signal enriched region, defined by $2.25<E^{*}<3.00 \mathrm{GeV}, 84 \pm 9$ events were found in real data with $91 \pm 4$ expected from simulation.

The stability of this result with respect to changing the selection criteria was studied. In particular, relaxing the $b$-tagging cut to 0.03 , as for the other analyses, gave $5 \pm 13$ signal events or $\operatorname{BR}(b \rightarrow s \gamma)=(1.0 \pm 2.5) \times 10^{-4}$ corresponding to an upper limit of $5.2 \times 10^{-4}$. Making the $b$-tagging requirement tighter by cutting at 0.001 gave an upper limit of $4.6 \times 10^{-4}$. The cut on the minimum energy of the photon candidates was moved to $5 \mathrm{GeV}$ and $8 \mathrm{GeV}$, this did not change the result of the fit.

In the region $2.25<\mathrm{E}^{*}<2.60 \mathrm{GeV}$, where the signal to background ratio is more favourable, the background is due mainly to $B \rightarrow D^{*} \pi^{0} X$ decays and $q \bar{q} \gamma$ events.

- The branching ratios for the two-body $B \rightarrow D^{*} \pi^{0}$ decays were tuned in the simulation in order to agree with the present world averages [30]. Their uncertainty was included in the systematic errors. The inclusive $\pi^{0}$ background was checked by repeating the analysis selecting $\pi^{0}$ candidates instead of photons. The shape of the $E^{*}$ distribution for real data was found to be reproduced by simulation. In the region $2.25<\mathrm{E}^{*}<2.60 \mathrm{GeV}$ there were $101 \pm 10$ events in real data compared with $106 \pm 5$ expected from simulation.

- The ratio of the number of $q \bar{q} \gamma$ events in real data to that in the simulation was estimated by a fit to the distribution of $E_{t} / E$ for the selected photons. The $q \bar{q} \gamma$ events are characterised by a broad distribution of $E_{t} / E$ extending to large values while other processes, including $b \rightarrow s \gamma$, are peaked at $E_{t} / E$ below 0.35 . An excess of events at $E_{t} / E$ above 0.45 was present in real data. From the result of a fit leaving the fraction of $q \bar{q} \gamma$ events free, the ratio of $q \bar{q} \gamma$ events in the data to that in the simulation was found to be compatible with 1.0 with an error of 0.25 . Changing this ratio from 1.00 to 1.25 would give $-7 \pm 14$ signal events, compatible with zero and thus with the present result. Lowering this ratio to 0.75 gives $5 \pm 14$ signal events corresponding to an upper limit of $5.7 \times 10^{-4}$.

The sensitivity of the upper limit to the predicted shape of the $E^{*}$ distribution for $b \rightarrow$ $s \gamma$ events was also studied. This was done by varying the value of $p_{F}$ from $0.27 \mathrm{GeV} / \mathrm{c}$, obtained from an analysis of the $B$ semi-leptonic decay [31], to $0.45 \mathrm{GeV} / \mathrm{c}$, which gives the best fit [27] to the photon spectrum obtained by CLEO. The change in the value of $p_{F}$ increases the smearing of the photon spectrum. The upper limit derived with the new value for $p_{F}$ increased to $5.0 \times 10^{-4}$.

To take the systematic errors into account, the levels of the $D^{(*)} \pi^{0}$ and $q \bar{q} \gamma$ backgrounds and the value of $p_{f}$ were varied as above and the resolution function, the absolute normalisation of real to simulated data, and the reconstruction efficiency were all 
changed by their uncertainties. The convolution of the changes in the fitted number of signal events was propagated to obtain the upper limit in the presence of systematic errors. The final result was:

$$
\mathrm{BR}(b \rightarrow s \gamma)<5.4 \times 10^{-4}
$$

This limit is compatible with the Standard Model expectations for $\operatorname{BR}(b \rightarrow s \gamma)$ and the results reported by the CLEO Collaboration.

\subsubsection{Exclusive searches}

For the $B_{d}^{0} \rightarrow K^{* 0} \gamma$ mode (Fig. 4), there are two events in the signal mass region with an expected background of $0.66 \pm 0.17$ events. This corresponds to a probability for a background fluctuation of 0.14 . Therefore no significant excess of events was observed and the upper limit on the number of signal events of 4.7 was derived at $90 \%$ confidence level corresponding to $\mathrm{BR}\left(B_{d}^{0} \rightarrow K^{*}(892)^{0} \gamma\right)<1.8 \times 10^{-4}$. The ratio of events in real and simulated data in the mass region from $3.5 \mathrm{GeV} / \mathrm{c}^{2}$ to $6.5 \mathrm{GeV} / \mathrm{c}^{2}$, excluding the signal region, was found to be $1.13 \pm 0.30$.

This result was cross-checked by performing a fit to the spectrum of the photon energy in the $B$ rest frame as was done for the inclusive $b \rightarrow s \gamma$ analysis. The fit gave $\operatorname{BR}\left(B_{d}^{0} \rightarrow\right.$ $\left.K^{*}(892)^{0} \gamma\right)=(7.8 \pm 6.8) \times 10^{-5}$, corresponding to an upper limit at $90 \%$ confidence level comparable with the one obtained above.

After including the effect of the systematic uncertainties on the background estimate and the reconstruction efficiency, the final result was:

$$
\operatorname{BR}\left(B_{d}^{0} \rightarrow K^{*}(892)^{0} \gamma\right)<2.1 \times 10^{-4}
$$

For the $B_{s}^{0} \rightarrow \phi \gamma$ mode, one candidate was selected with an estimated background of $0.35 \pm 0.13$ events. Thus no excess of events was seen and the upper limit for the decay branching ratio was found to be:

$$
\operatorname{BR}\left(B_{s}^{0} \rightarrow \phi \gamma\right)<7.0 \times 10^{-4}
$$

at $90 \%$ confidence level and including systematic uncertainties (Table 7 ).

\section{Dineutrino charmless decays}

Like the radiative $b \rightarrow s \gamma$ decays, $b \rightarrow s l \bar{l}$ decays with $l=e, \mu, \nu$ have also received considerable theoretical attention [20,33,34]. In the Standard Model, dineutrino decays $b \rightarrow s \nu \bar{\nu}$ (Fig. 1e) are simpler to treat than other classes of rare decays involving dileptons, such as $b \rightarrow s \mu^{+} \mu^{-}$, and therefore the rates predicted are subject to smaller uncertainties. The estimated rate for $b \rightarrow s \nu \bar{\nu}$ is in the range $(0.4-1.0) \times 10^{-4}$ for $m_{\text {top }}=180 \mathrm{GeV} / \mathrm{c}^{2}$, with about $30 \%$ of the inclusive rate going through $B \rightarrow K^{*} \nu \bar{\nu}[34,35]$. Analogously to $b \rightarrow s \gamma$ decays, the rate for this decay can be modified by the contribution of new particles in the loop.

In addition it has recently been pointed out [36] that a new $U(1)$ gauge boson $X$, coupling predominantly to third family fermions, could give a large increase of the rate $b \rightarrow s \nu_{\tau} \bar{\nu}_{\tau}$ produced by tree level $X$ boson exchange in addition to $Z$ exchange. For specific combinations of the $X$ boson mass $M_{X}$, its coupling $g_{X}$ and the $Z-X$ mixing angle, this rate can become as large as the $b$ semi-leptonic decay rate.

At LEP this process can be searched for in exclusive decays consisting of a secondary strange particle accompanied by large missing energy due to the presence of the two 
neutrinos. At LEP energies, the decay products of the two $b$ quarks are contained in opposite hemispheres. This is essential for tagging the presence of a beauty hadron decaying into $s \nu \bar{\nu}$ using the missing energy. To suppress the large backgrounds from partially reconstructed $s, c$ and $b$ decays, the analysis was performed using the exclusive final states $K^{*} \nu \bar{\nu}$ and $\phi \nu \bar{\nu}$. The cuts on the invariant mass of the strange mesons and the secondary vertex reconstruction reduce the combinatorial and other backgrounds.

\subsection{Event reconstruction}

Hadronic events satisfying the $b$-tagging criteria were selected as in the two previous analysis. The $b \rightarrow s \nu \bar{\nu}$ decays were searched for in the exclusive channels:

- $B_{d}^{0} \rightarrow K^{*}(892)^{0} \nu \bar{\nu}, \quad K^{*}(892)^{0} \rightarrow K^{+} \pi^{-}$

- $B_{s}^{0} \rightarrow \phi(1020) \nu \bar{\nu}, \quad \phi(1020) \rightarrow K^{+} K^{-}$.

The reconstruction started with an identified charged $K$ having momentum larger than $3.5 \mathrm{GeV} / \mathrm{c}$. Oppositely charged particles belonging to the same jet and having momentum above $1 \mathrm{GeV} / \mathrm{c}$ were tested for forming a common vertex with the charged kaon. Vertices with a low fit probability or a decay distance with respect to the primary vertex normalized to its error below 2.5 were rejected.

The characteristics of the $B \rightarrow K^{*}(\phi) \nu \bar{\nu}$ signals were studied on fully simulated events. For this simulation the JETSET event generator was used and events were reweighted in order to reproduce the predicted mass distribution of the $\nu \bar{\nu}$ system [34]. Signal $b \rightarrow s \nu \bar{\nu}$ events can be separated from most of the background sources by using the energy detected in the hemisphere. The visible energy $E_{v i s}$ in the hemisphere containing the strange meson candidate was determined as the sum of the energy in charged particles $E_{c h a}$, in electromagnetic showers measured in the HPC calorimeter $E_{H P C}$, and in neutral hadrons detected by the hadron calorimeter $E_{H C A L}$. The missing energy was defined as $E_{\text {miss }}=E_{\text {hem }}-E_{\text {vis }}$. The total energy in the hemisphere $E_{\text {hem }}$ was determined imposing four-momentum conservation and it is given by $E_{\text {hem }}=E_{\text {beam }}-\left(M_{o h}^{2}-M_{s h}^{2}\right) /\left(4 E_{\text {beam }}\right)$ where $E_{\text {beam }}$ is the beam energy and $M_{s h}, M_{o h}$ the invariant mass of the same and of the opposite hemisphere with respect to the strange particle candidate. The resolution on the missing energy can be parametrised by a Gaussian distribution with a resolution $\sigma$ of $5 \mathrm{GeV}$ and a wider component extending to larger values of missing energy.

Signal events are characterized by a large missing energy corresponding to a low value of $E_{\text {vis }} / E_{h e m}$ and a large fraction of the energy in charged hadrons and photons taken by the strange meson candidate. Semi-leptonic decays of either $b$ or $c$ quarks can also give large missing energy due to the emission of a neutrino and therefore represent a potential source of background. These events were removed by rejecting all $K^{+} \pi^{-}$and $K^{+} K^{-}$ pairs having a tagged lepton in the same hemisphere. Events for which the missing momentum vector points outside the barrel region were rejected since the missing energy is likely to be due to neutrals outside the acceptance of the calorimeters. Events were also rejected if the invariant mass of the hemisphere containing the strange meson was above $10 \mathrm{GeV} / c^{2}$ since signal $b \rightarrow s \nu \bar{\nu}$ events are characterised by a low jet mass due to the missing neutrinos.

\subsection{Results}

To separate possible signal candidates from the bulk of the background, the variable $\alpha$ describing the position of each selected entry in the $E_{K^{*}} /\left(E_{c h a}+E_{H P C}\right), E_{v i s} / E_{h e m}$ 
plane was defined (Fig. 5). For the events in the signal enriched region corresponding to $\alpha$ below 0.9, a binned likelihood fit to the $K \pi(K K)$ invariant mass distribution was performed to extract the number of events containing a $K^{*}$ or $\phi$ strange meson resonance.

In real data $70 \pm 18(97 \pm 16)$ events with a $K^{*}(\phi)$ were seen with an expected background of $K^{*}(\phi)$ from other processes of $76 \pm 7$ (94 \pm 6 ) (Fig. 6). This corresponds to $90 \%$ confidence level upper limits for the number of signal events of 32 and 30 . A study of the simulated signal sample showed that $(9.0 \pm 0.7) \%$ of $K^{*} \nu \bar{\nu}$ signal events and $(7 \pm 1) \%$ of $\phi \nu \bar{\nu}$ satisfy these selection criteria. The following upper limits were obtained from these numbers: $B R\left(B_{d} \rightarrow K^{*} \nu \bar{\nu}\right)<9.5 \times 10^{-4}$ and $B R\left(B_{s} \rightarrow \phi \nu \bar{\nu}\right)<4.9 \times 10^{-3}$.

The agreement between the data and the Monte Carlo in describing the background was verified. The ratios of the events in real data and simulation in the region of $\alpha$ above 0.9 is $1.02 \pm 0.03(0.95 \pm 0.05)$, showing that the rejection factors of the selection cuts for real data are well reproduced by the simulation. Also the number of $K^{*}$ and $\phi$ candidates obtained from the fit to the mass distributions in real data and simulation were found to be in agreement. Before applying the cut on the angular variable $\alpha$, the ratios of these numbers were $1.00 \pm 0.07$ and $0.98 \pm 0.07$ for $K^{*}$ and $\phi$ mesons respectively. Including the statistical errors of these comparisons as contributions to the systematic uncertainty, the final values of the upper limits are (Table 8 ):

$$
B R\left(B_{d} \rightarrow K^{*} \nu \bar{\nu}\right)<1.0 \times 10^{-3}
$$

and

$$
B R\left(B_{s} \rightarrow \phi \nu \bar{\nu}\right)<5.4 \times 10^{-3} .
$$

These limits place a constraint on the combination $\left(g_{X} / M_{X}\right)^{2} \times$ mixing angle in an extended theory with a new $U(1)$ gauge boson $X$ coupling predominantly to the third generation of fermions [36]. Assuming a ratio between the exclusive $B \rightarrow K^{*} \nu \bar{\nu}$ branching ratio and the inclusive $b \rightarrow s \nu \bar{\nu}$ rate of 0.30 , an upper bound for the mixing angle $\left|\lambda_{23}^{L}\right|^{2}+\left|\lambda_{23}^{R}\right|^{2}$ follows from the upper limit obtained for $B^{0} \rightarrow K^{*} \nu \bar{\nu}$. If $\left(g_{X} / M_{X}\right)^{2}=$ $G / 2 \sqrt{2}$, the upper bound is $1.0 \times\left|V_{c b}\right|$. Taking $\left(M_{X} / g_{X}\right)=1 \mathrm{TeV}$ the limit becomes 4.0 $\times\left|V_{c b}\right|$.

\section{Conclusions}

A search for rare decays of the $b$ quark in charmless hadronic, radiative and dineutrino modes has been performed using $3 \times 10^{6} Z^{0}$ hadronic decays recorded by the DELPHI detector at LEP.

Evidence for charmless hadronic decays of $B$ mesons was obtained by observing eight events in two-body modes. The branching ratio for $B_{d, s}^{0} \rightarrow\left(\pi^{+} \pi^{-}+K^{+} \pi^{-}\right)$was estimated to be $\left(2.8_{-1.0}^{+1.5}\right.$ (stat.) \pm 0.2 (syst.) $) \times 10^{-5}$ from five events and that for $B_{u}^{-} \rightarrow(\rho \pi$ $\left.+K^{*} \pi\right)$ was estimated to be $\left(1.7_{-0.8}^{+1.2}\right.$ (stat.) \pm 0.2 (syst.) $) \times 10^{-4}$ from three events. The exclusive decay $B_{d, s}^{0} \rightarrow K^{+} \pi^{-}$was observed with a rate of $\left(2.4_{-1.1}^{+1.7}\right.$ (stat.) \pm 0.2 (syst.) $) \times 10^{-5}$ from three events. In each case, the probability of the observed signal having arisen from a background fluctuation was below $10^{-3}$.

The fraction of these hadronic charmless $b$ decays with a kaon in the final state not due to a spectator $s$ quark was also measured. It was found to be $0.58 \pm 0.18$. This value agrees with the expectation if the $b \rightarrow s$ and the $b \rightarrow u$ decay processes contribute almost equally. The same fit gave $1.3_{-1.3}^{+1.5}$ for the number of $B_{s}^{0}$ candidates in the sample, to be compared with 1.5 expected assuming the $B_{s} / B_{d}$ production ratio $f_{s} / f_{d}=0.30$. 
Improved upper limits were set for other two-body hadronic charmless $B$ meson decays and for the charmless decay of the beauty baryon $\Lambda_{b} \rightarrow p K^{-}$(see Table 4), and also for three body (Table 5) and four body (Table 6) $B$ meson decays.

Using an inclusive algorithm for reconstructing the hadronic system accompanying an energetic photon, candidate $b \rightarrow s \gamma$ events were separated from the dominant $b \rightarrow c$ background. No excess of events was found in the signal region. An upper limit for $\mathrm{BR}(b \rightarrow s \gamma)$ of $5.4 \times 10^{-4}$ at $90 \%$ confidence level was obtained.

The exclusive decays $B_{d}^{0} \rightarrow K^{*}(892)^{0} \gamma$ and $B_{s}^{0} \rightarrow \phi \gamma$ were excluded at $90 \%$ confidence level for branching ratios above $2.1 \times 10^{-4}$ and $7.0 \times 10^{-4}$ respectively.

Finally the first limits for the exclusive charmless dineutrino decays $B_{d}^{0} \rightarrow K^{*} \nu \bar{\nu}$ and $B_{s}^{0} \rightarrow \phi \nu \bar{\nu}$ were found to be less than $1.0 \times 10^{-3}$ and $5.4 \times 10^{-3}$ at $90 \%$ confidence level. These limits have implications on models with an additional $U(1)$ gauge boson coupling predominantly to the third family.

\section{Acknowledgements}

We are greatly indebted to our technical collaborators and to the funding agencies for their support in building and operating the DELPHI detector, and to the members of the CERN-SL Division for the excellent performance of the LEP collider. 


\section{References}

[1] W. Hou, Nucl. Phys. B 308 (1988) 561.

[2] D. London and R. Peccei, Phys. Lett. B 223 (1989) 257;

M. Gronau, Phys. Lett. B 300 (1993) 163.

[3] P. Aarnio et al., Nucl. Instr. Meth. A 303 (1991) 233.

[4] P. Abreu et al., CERN-PPE/95-194, to be published in Nucl. Instr. Meth. A.

[5] W. Adam et al., in Proceedings of the 1995 Int. Workshop on Ring Imaging Cherenkov Detectors, to be published in Nucl. Instr. and Meth. A.

[6] V. Chabaud et al., Nucl. Instr. Meth. A 368 (1996) 314.

[7] P. Abreu et al., Phys. Lett. B 312 (1993) 253.

[8] P. Abreu et al., Z. Phys. C 66 (1995) 323.

[9] M. Battle et al., Phys. Rev. Lett. 71 (1993) 3922.

[10] R. Akers et al., Phys. Lett. B 337 (1994) 393.

[11] P. Abreu et al., Phys. Lett. B 357 (1995) 255.

[12] M. Acciarri et al., Phys. Lett. B 363 (1995) 127.

[13] J. Boucrot et al., EPS-0399, contributed paper to the EPS 1995 conference, Brussels, July 1995.

[14] L.L. Chau et al., Phys. Rev. D 43 (1991) 2176.

[15] N.G. Deshpande and J. Trampetic, Phys. Rev. D 41 (1990) 895.

[16] A. Deandrea et al., Phys. Lett. B 320 (1994) 170.

[17] N.G. Deshpande and X.G. He, Phys. Lett. B 336 (1994) 471.

[18] K. Berkelman, Hadronic Decays, in B Decays ed. by S. Stone, World Scientific, Singapore 1992.

[19] M. Ciuchini et al., Phys.Lett. B 334 (1994) 137.

[20] S. Bertolini, F. Borzumati, A. Masiero and G. Ridolfi, Nucl. Phys. B 353 (1991) 591.

[21] V. Barger, M.S. Berger and R.J.N. Phillips, Phys. Rev. Lett. 70 (1993) 1368;

R. Garisto and J.N. Ng, Phys. Lett. B 315 (1993) 372.

[22] R. Ammar et al., Phys. Rev. Lett. 71 (1993) 674.

[23] M.S. Alam et al., Phys. Rev. Lett. 74 (1995) 2885.

[24] M. Battaglia, in Proceedings of the $5^{\text {th }}$ Int. Symp. on Heavy Flavour Physics, edited by D.I. Britton, D.B. MacFarlane and P.M. Patel, Edition Frontieres, 1993.

[25] O. Adriani et al., Phys. Lett. B 317 (1993) 637.

[26] A. Ali and C. Greub, Phys. Lett. B 259 (1991) 182.

[27] A. Ali and C. Greub, Phys. Lett. B 361 (1995) 146.

[28] A. Ali et al., Phys.Lett. B 298 (1993) 195.

[29] T. Sjöstrand, Comp. Phys. Comm. 82 (1994) 74.

[30] Particle Data Group, Phys. Rev. D 50 (1994).

[31] M.S. Alam et al., Phys. Rev. Lett. 74 (1995) 2885.

[32] A. Ali, W. Braun and H. Simma, Z. Phys. C63 (1994) 437.

[33] N.G. Deshpande and J. Trampetic, Phys. Rev. Lett. 60 (1988) 2583.

[34] A. Ali and T. Mannel., Phys. Lett. B 264 (1991) 447.

[35] G. Buchalla and A.J. Buras, Nucl. Phys. B 400 (1993) 225.

[36] B. Holdom and M.V. Ramana, Phys.Lett. B 365 (1996) 309. 
Table 1: Characteristics of the candidate events in two-body decay modes. The invariant mass, energy $E_{B}$, decay distance in units of significance $d / \sigma_{d}$, and proper decay time $\tau_{B}$ For the $K^{*} \pi$ and $\rho \pi$ candidates, the value of $\left|\cos \theta^{*}\right|$, where $\theta^{*}$ is the helicity angle between the direction of the $K$ or $\pi$ from the resonance decay and the $B$ direction in the resonance rest frame, and the distance of the measured resonance mass from its central value in $\sigma$ units are also given. The first $\pi \pi$ event is ambiguous with the $K \pi$ hypothesis.

\begin{tabular}{|c|c|c|c|c|c|c|}
\hline Channel & $\begin{array}{c}\text { Mass } \\
{\left[\mathrm{GeV} / c^{2}\right]}\end{array}$ & $\begin{array}{c}E_{B} \\
{[\mathrm{GeV}]}\end{array}$ & $d / \sigma_{d}$ & $\begin{array}{c}\tau_{B} \\
{[\mathrm{ps}]}\end{array}$ & $\left|\cos \theta^{*}\right|$ & $\begin{array}{c}\text { Distance in } \sigma \\
\text { from resonant mass }\end{array}$ \\
\hline \hline$\pi \pi$ & $5.18 \pm 0.11$ & 23.0 & 6.4 & 3.1 & - & - \\
\hline$\pi \pi$ & $5.24 \pm 0.07$ & 21.2 & 6.4 & 2.6 & - & - \\
\hline$K \pi$ & $5.19 \pm 0.08$ & 27.5 & 3.2 & 0.3 & - & - \\
\hline$K \pi$ & $5.20 \pm 0.08$ & 39.3 & 3.0 & 0.3 & - & - \\
\hline$K \pi$ & $5.47 \pm 0.10$ & 43.7 & 8.5 & 1.5 & - & - \\
\hline$\rho \pi$ & $5.34 \pm 0.09$ & 42.0 & 70.4 & 3.4 & 0.68 & +0.45 \\
\hline$K^{*} \pi$ & $5.21 \pm 0.06$ & 40.0 & 17.6 & 1.2 & 0.59 & +0.78 \\
\hline$K^{*} \pi$ & $5.38 \pm 0.07$ & 39.0 & 44.6 & 2.3 & 0.63 & +1.60 \\
\hline
\end{tabular}

Table 2: Characteristics of the candidate events in three-body decay modes.

\begin{tabular}{|c|c|c|c|c|}
\hline Channel & $\begin{array}{c}\text { Mass } \\
{\left[\mathrm{GeV} / c^{2}\right]}\end{array}$ & $\begin{array}{c}E_{B} \\
{[\mathrm{GeV}]}\end{array}$ & $d / \sigma_{d}$ & $\begin{array}{c}\tau_{B} \\
{[\mathrm{ps}]}\end{array}$ \\
\hline \hline$\pi \pi \pi$ & $5.26 \pm 0.05$ & 34.6 & 27.2 & 2.1 \\
\hline$K \pi \pi$ & $5.23 \pm 0.05$ & 40.8 & 5.1 & 2.4 \\
\hline$K \pi \pi$ & $5.39 \pm 0.04$ & 20.3 & 17.6 & 3.0 \\
\hline$K \pi \pi$ & $5.27 \pm 0.06$ & 40.2 & 11.6 & 1.0 \\
\hline$K \pi \pi$ & $5.32 \pm 0.04$ & 21.6 & 11.8 & 1.2 \\
\hline
\end{tabular}

Table 3: Characteristics of the candidate events in four-body decay modes.

\begin{tabular}{|c|c|c|c|c|}
\hline Channel & $\begin{array}{c}\text { Mass } \\
{\left[\mathrm{GeV} / c^{2}\right]}\end{array}$ & $\begin{array}{c}E_{B} \\
{[\mathrm{GeV}]}\end{array}$ & $d / \sigma_{d}$ & $\begin{array}{c}\tau_{B} \\
{[\mathrm{ps}]}\end{array}$ \\
\hline \hline$\pi \pi \pi \pi$ & $5.43 \pm 0.05$ & 39.6 & 13.2 & 1.4 \\
\hline$\pi \pi \pi \pi$ & $5.21 \pm 0.05$ & 41.4 & 16.8 & 0.9 \\
\hline$K \pi \pi \pi$ & $5.23 \pm 0.11$ & 44.5 & 46.0 & 0.3 \\
\hline
\end{tabular}


Table 4: Summary of the results for two-body decays giving the number of candidates in each channel, the estimated background, the reconstruction efficiency, and the corresponding result for the decay branching ratio and its comparison with theoretical predictions. All upper limits are computed at $90 \%$ confidence level.

\begin{tabular}{|l|c|c|c|c|c|c|c|}
\hline Channel & Evts & Bkg & $\begin{array}{c}\epsilon \\
{[\%]}\end{array}$ & $\begin{array}{c}\text { Signal } \\
\text { Evts }\end{array}$ & \multicolumn{2}{|c|}{$\begin{array}{c}\text { Theory } \\
\text { BR } \times 10^{5}\end{array}$} & $\begin{array}{c}\text { DELPHI } \\
\text { BR } \times 10^{5}\end{array}$ \\
\hline \hline$B_{d}^{0} \rightarrow \pi^{+} \pi^{-}$ & 2 & 0.09 & 23.0 & $<5.2$ & $1.1-1.8$ & {$[14,16]$} & $<4.5$ \\
$B_{d, s}^{0} \rightarrow K^{+} \pi^{-}$ & 3 & 0.06 & 18.0 & 3 & $1.1-1.8$ & {$[14-16]$} & $2.4_{-1.1}^{+1.7} \pm 0.2$ \\
$B_{d, s}^{0} \rightarrow \pi^{+} \pi^{-}, K^{+} \pi^{-}$ & 5 & 0.15 & 25.0 & 5 & - & & $2.8_{-1.0}^{+1.5} \pm 0.2$ \\
$B_{d, s}^{0} \rightarrow K^{+} K^{-}$ & 0 & 0.01 & 8.0 & $<2.3$ & - & & $<4.6$ \\
$\Lambda_{b}^{0} \rightarrow p K^{-}$ & 0 & 0.01 & 5.5 & $<2.3$ & - & & $<36$ \\
$B_{u}^{-} \rightarrow \rho^{0} \pi^{-}$ & 1 & 0.09 & 5.5 & $<3.8$ & $0.4-1.4$ & {$[14,16]$} & $<16$ \\
$B_{u}^{-} \rightarrow K^{* 0} \pi^{-}$ & 2 & 0.06 & 4.5 & $<5.3$ & $0.6-0.9$ & {$[14,15]$} & $<39$ \\
$B_{u}^{-} \rightarrow \rho^{0} \pi^{-}, K^{* 0} \pi^{-}$ & 3 & 0.15 & 6.0 & 3 & - & & $17_{-}^{+12} \pm 2$ \\
$B_{u}^{-} \rightarrow K^{-} \rho^{0}$ & 0 & 0.17 & 4.5 & $<2.3$ & $0.01-0.06$ & {$[14,15]$} & $<12$ \\
$B_{u}^{-} \rightarrow K^{-} \phi$ & 0 & 0.01 & 4.0 & $<2.3$ & $0.6-1.4$ & {$[14,15,17]$} & $<28$ \\
$B_{d, s}^{0} \rightarrow K^{+} a_{1}^{-}$ & 0 & 0.18 & 3.5 & $<2.3$ & - & & $<23$ \\
\hline
\end{tabular}

Table 5: Summary of the $90 \%$ confidence level upper limits for three-body decays.

\begin{tabular}{|c|c|c|c|c|c|c|}
\hline Channel & Evts & Bkg. & $\begin{array}{c}\epsilon \\
\%\end{array}$ & $\begin{array}{c}\text { Signal } \\
\text { Event UL }\end{array}$ & $\begin{array}{c}\text { Theory } \\
\text { BR } \times 10^{5}\end{array}$ & $\begin{array}{l}\text { DELPHI } \\
\mathrm{BR} \times 10^{5}\end{array}$ \\
\hline$B_{u}^{-} \rightarrow \pi^{+} \pi^{-} \pi^{-}$ & 1 & 1.9 & 5.3 & $<3.0$ & \begin{tabular}{l|l}
6 & {$[18]$}
\end{tabular} & $<13$ \\
\hline$B_{u}^{-} \rightarrow K^{-} \pi^{+} \pi^{-}$ & 4 & 1.6 & 4.3 & $<6.4$ & - & $<33$ \\
\hline$B_{u}^{-} \rightarrow K^{+} K^{-} K^{-}$ & 0 & 0.03 & 2.6 & $<2.3$ & - & $<20$ \\
\hline
\end{tabular}

Table 6: Summary of the $90 \%$ confidence level limits for four-body decays.

\begin{tabular}{|c|c|c|c|c|c|c|}
\hline Channel & Evts & Bkg. & $\begin{array}{c}\epsilon \\
{[\%]}\end{array}$ & $\begin{array}{c}\text { Signal } \\
\text { Event UL }\end{array}$ & $\begin{array}{c}\text { Theory } \\
\text { BR } \times 10^{5}\end{array}$ & $\begin{array}{l}\text { DELPHI } \\
\mathrm{BR} \times 10^{5}\end{array}$ \\
\hline$B_{d}^{0} \rightarrow \pi^{+} \pi^{+} \pi^{-} \pi^{-}$ & 2 & 2.9 & 3.8 & $<3.5$ & [18] & $<23$ \\
\hline$B_{d, s}^{0} \rightarrow K^{+} \pi^{+} \pi^{-} \pi^{-}$ & 1 & 2.4 & 2.3 & $<2.9$ & - & $<23$ \\
\hline
\end{tabular}


Table 7: Summary of the $90 \%$ confidence level upper limits for radiative decays.

\begin{tabular}{|l|c|c|c|c|c|c|c|}
\hline Channel & Evts & Bkg. & $\begin{array}{c}\epsilon \\
{[\%]}\end{array}$ & $\begin{array}{c}\text { Signal } \\
\text { Event UL }\end{array}$ & \multicolumn{2}{|c|}{$\begin{array}{c}\text { Theory } \\
\mathrm{BR} \times 10^{5}\end{array}$} & $\begin{array}{c}\text { DELPHI } \\
\mathrm{BR} \times 10^{5}\end{array}$ \\
\hline \hline$b \rightarrow s \gamma$ & 84 & 91 & 0.042 & $<23$ & $19 \pm 5$ & {$[19]$} & $<54$ \\
$B_{d}^{0} \rightarrow K^{* 0} \gamma$ & 2 & 0.67 & 0.076 & $<4.7$ & $1.0-11.0$ & {$[19,28]$} & $<21$ \\
$B_{s}^{0} \rightarrow \phi \gamma$ & 1 & 0.35 & 0.075 & $<2.3$ & $1.0-11.0$ & {$[19,28,32]$} & $<70$ \\
\hline
\end{tabular}

Table 8: Summary of the $90 \%$ confidence level upper limits for dineutrino decays.

\begin{tabular}{|c|c|c|c|c|c|c|c|}
\hline Channel & Evts & Bkg. & $\begin{array}{c}\epsilon \\
{[\%]}\end{array}$ & $\begin{array}{c}\text { Signal } \\
\text { Event UL }\end{array}$ & $\begin{array}{l}\text { The } \\
\text { BR }\end{array}$ & $\begin{array}{l}\text { ory } \\
<10^{5} \\
\end{array}$ & $\begin{array}{l}\text { DELPHI } \\
\mathrm{BR} \times 10^{5}\end{array}$ \\
\hline$B_{d}^{0} \rightarrow K^{* 0} \nu \bar{\nu}$ & 70 & 76 & 0.09 & $<32$ & $1.0-3.0$ & {$[34,35]$} & $<100$ \\
\hline$B_{s}^{0} \rightarrow \phi \nu \bar{\nu}$ & 97 & 94 & 0.07 & $<30$ & - & & $<540$ \\
\hline
\end{tabular}



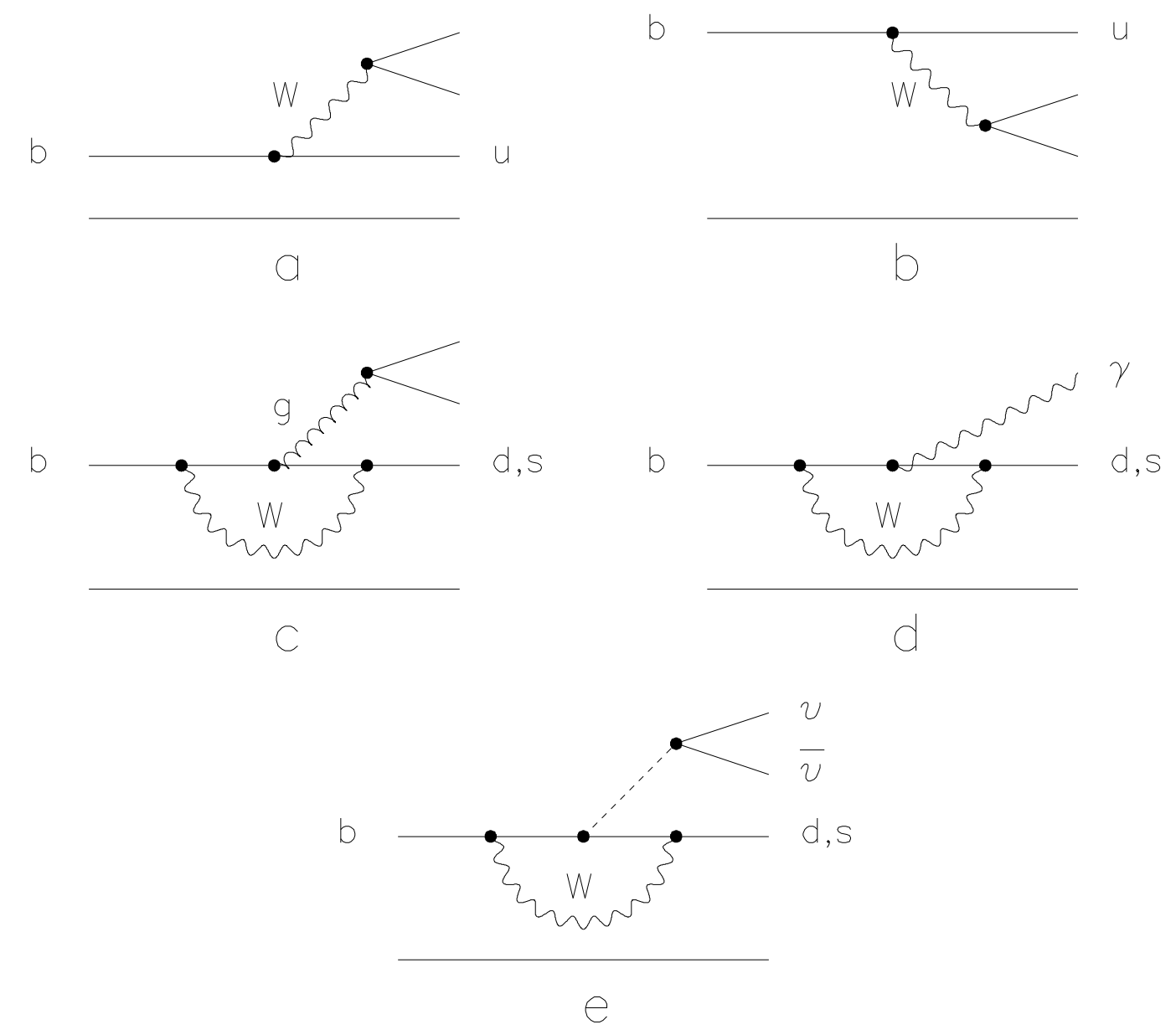

Figure 1: Feynman diagrams of the main processes contributing to the rare $b$ decays studied in the present paper: hadronic charmless $b$ decays through tree level diagrams (a and $b$ ) and penguin diagrams (c), and radiative $(d)$ and dineutrino (e) decays also through penguin diagrams 


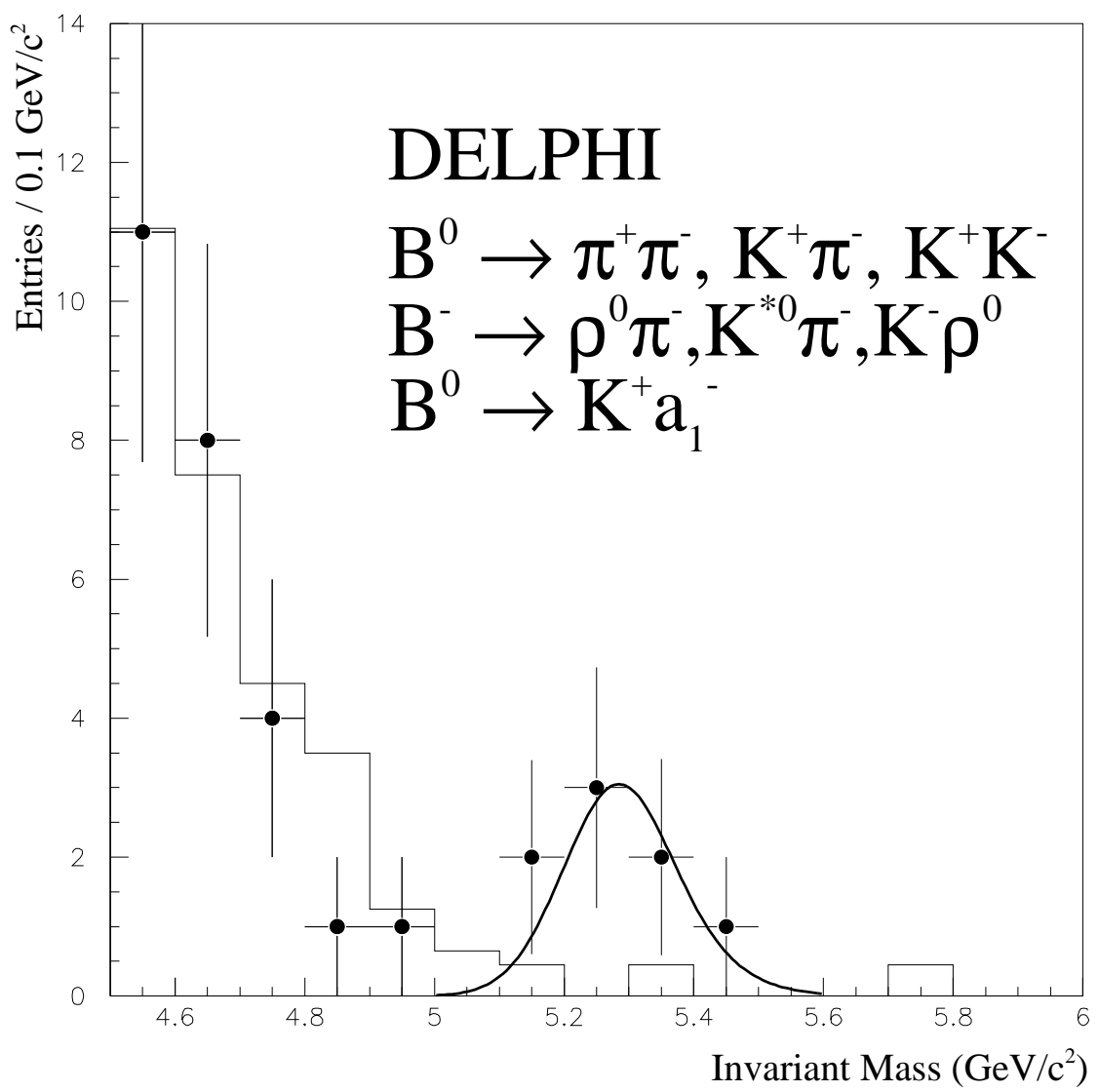

Figure 2: Invariant mass distribution for two body charmless hadronic $B$ decay channels. The points with error bars represent the real data and the histograms the mass distributions expected in the absence of charmless hadronic $B$ decays, as obtained from simulation. The curve represents the shape expected for the signal events normalised to the number of candidates selected in real data in the signal mass region. 

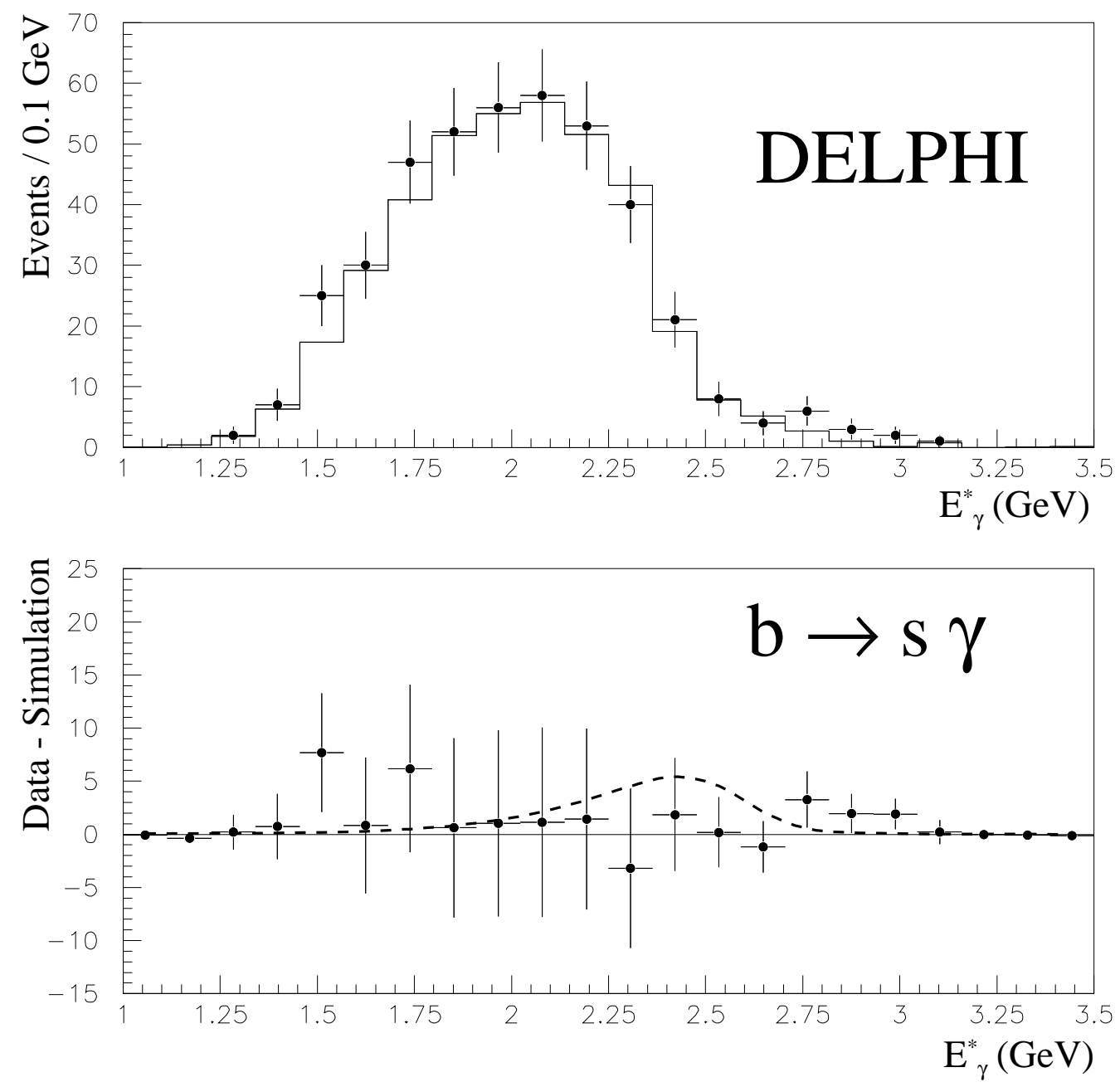

Figure 3: Energy spectrum of the photons selected in the inclusive $b \rightarrow s \gamma$ analysis in the rest frame of the $B$ meson. Real data and background from simulation are shown in the upper plot. The data points after background subtraction are shown in the lower plot, where the dashed curve corresponds to the $90 \%$ confidence level upper limit obtained from the fit. 


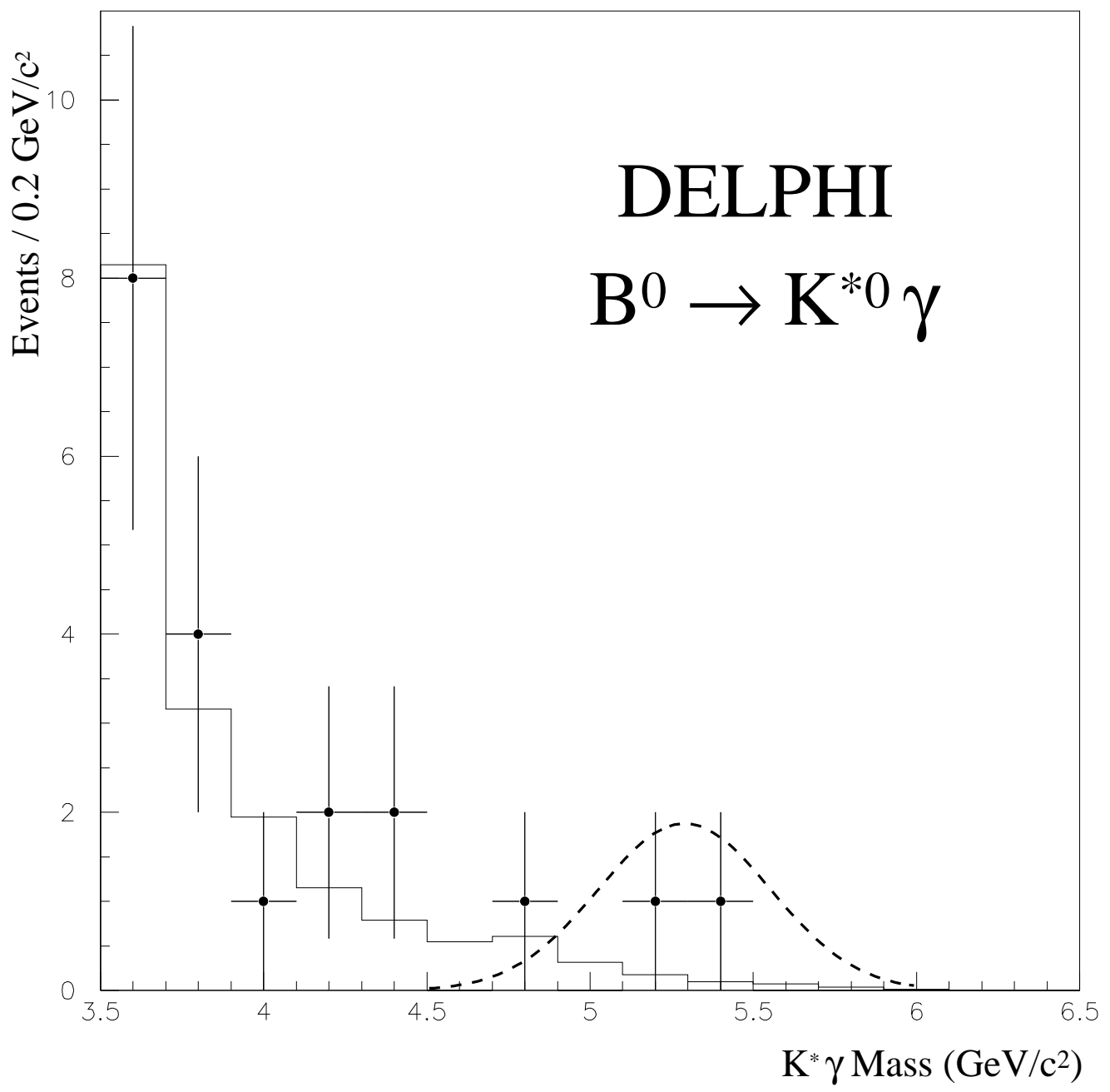

Figure 4: The mass distribution for the $\left(K^{+} \pi^{-}\right)_{K^{* 0}} \gamma$ system. Points with error bars represent the real data and the histograms the expected mass distributions in the absence of charmless radiative $B$ decays, as obtained from simulation. The dashed curve represents the expected $B^{0} \rightarrow K^{* 0} \gamma$ signal corresponding to the $90 \%$ upper limit quoted. 

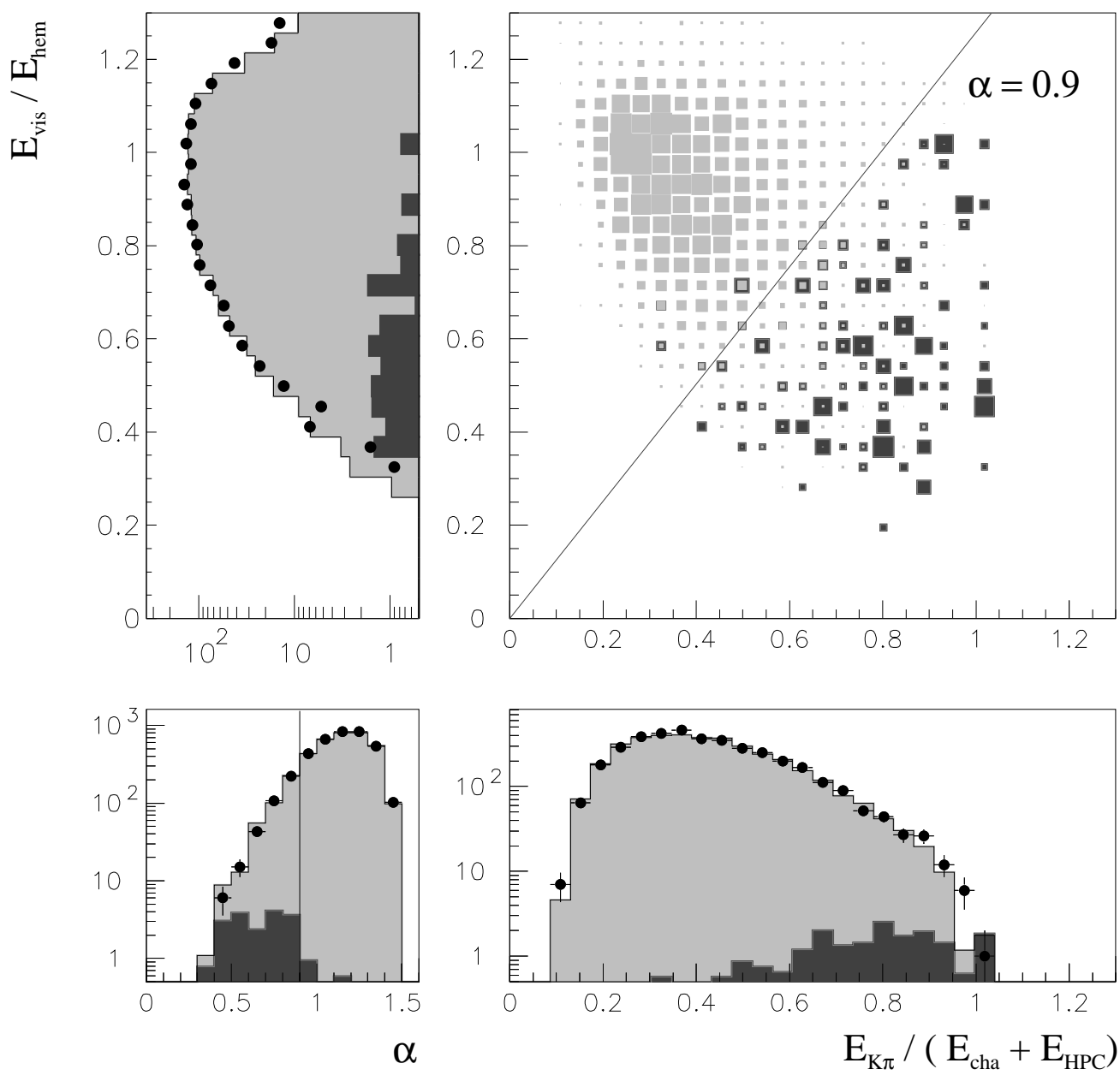

Figure 5: Distributions for the $E_{\text {vis }} / E_{h e m}$ (upper left) and $E_{K \pi} /\left(E_{c h a}+E_{H P C}\right)$ (lower right) variables used in the search for dineutrino charmless $B$ decays and their correlation (upper right). The angular variable $\alpha$ used to select the signal enriched region is shown in the lower left plot. The cut $\alpha=0.9$ is also shown (upper right and lower left). Real data are shown by the closed circles, the background from simulation by the light grey histograms, and the distributions expected for signal $B \rightarrow K^{* 0} \nu \bar{\nu}$ events by the dark grey histograms. 

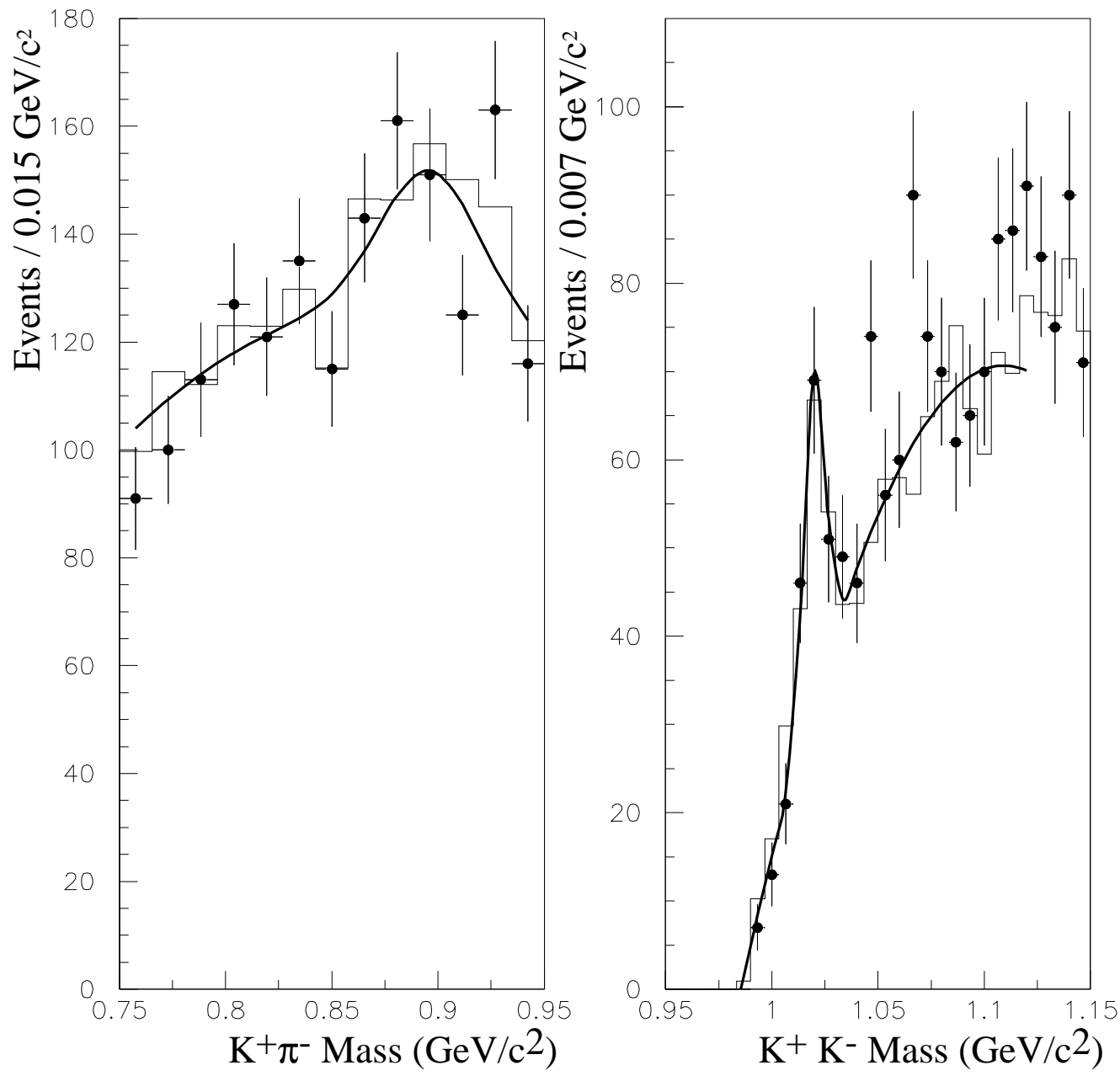

Figure 6: The invariant mass distribution for $K^{+} \pi^{-}$(left) and $K^{+} K^{-}$(right) pairs for events in the signal $b \rightarrow s \nu \bar{\nu}$ region of $\alpha$ below 0.9 . The points with error bars show the distribution for real data and the histogram the one for simulation. The curves show the fits to the distributions used to extract the numbers of $K^{*}$ and $\phi$ mesons. 